TRANSACTIONS OF THE

AMERICAN MATHEMATICAL SOCIETY

Volume 359, Number 1, January 2007, Pages 385-415

S 0002-9947(06)03884-0

Article electronically published on August 16, 2006

\title{
KOSZUL DUALITY FOR TORIC VARIETIES
}

\author{
TOM BRADEN
}

\begin{abstract}
We show that certain categories of perverse sheaves on affine toric varieties $X_{\sigma}$ and $X_{\sigma} \vee$ defined by dual cones are Koszul dual in the sense of Beilinson, Ginzburg and Soergel (1996). The functor expressing this duality is constructed explicitly by using a combinatorial model for mixed sheaves on toric varieties.
\end{abstract}

\section{INTRODUCTION}

In BGS Beilinson, Ginzburg, and Soergel described a remarkable duality on the category of highest-weight modules for a semisimple complex Lie algebra $\mathfrak{g}$. Expressed algebraically, it says that the ring governing this abelian category, endowed with a certain grading, is Koszul self-dual. Koszul duality is a relation between graded rings which generalizes the duality between symmetric and exterior algebras.

By localizing highest weight modules, this can be expressed as a duality on the category of Bruhat-constructible (mixed) perverse sheaves on the flag variety associated to $\mathfrak{g}$. However, the methods of [BGS] do not completely explain how Koszul duality might arise from geometry. In fact, no combination of standard functors such as $R j^{*}$ and $j^{!}$can have the right effect on the mixed structure.

In this paper we construct an analogous Koszul duality between categories of perverse sheaves on a pair of affine toric varieties defined by dual cones. Our proof is ultimately combinatorial, but it is more "local" than the proof of [BGS], and we hope that our methods will eventually lead to a more geometric understanding of Koszul duality.

Although the singularities of toric varieties are in many ways simpler than the ones appearing in the Bruhat stratification, the two situations satisfy very similar combinatorics. For instance, Stanley $\mathrm{St}$ ] proved a convolution identity for $g$-polynomials of convex polytopes (which measure local intersection cohomology of toric varieties) in which both a polytope $P$ and its polar $P^{\vee}$ appeared, along with their faces. This identity is exactly analogous to an identity for Kazhdan-Lusztig polynomials from $\mathrm{KL}$. As was noted in [BGS], Koszul duality for highest weight modules can be seen as a categorical lifting of the Kazhdan-Lusztig identity. This was our original motivation to conjecture that there would be a similar duality for toric varieties.

The appearance of dual cones and the strange interaction with (mixed) Hodge structures suggests that there should be some relation with mirror symmetry. We

Received by the editors March 23, 2004 and, in revised form, November 20, 2004.

2000 Mathematics Subject Classification. Primary 14M25, 16S37, 55N33, 18F20.

This work was supported in part by NSF grant DMS-0201823.

(C)2006 American Mathematical Society Reverts to public domain 28 years from publication 
do not yet understand what such a relation might be, but we remark that Stanley's identity was used in $\mathrm{BaBo}$ in the proof of the equality of stringy Hodge numbers for mirror dual toric hypersurfaces.

1.1. Main result, first version. Let $V$ be an $n$-dimensional real vector space, spanned by a lattice $V_{\mathbb{Z}} \subset V$. Let $\sigma \subset V$ be an $n$-dimensional pointed polyhedral cone, rational with respect to $V_{\mathbb{Z}}$. We denote by $[\sigma]$ the poset (fan) of faces of $\sigma$, with order relation $\prec$ given by inclusion of faces.

The dual cone $\sigma^{\vee} \subset V^{*}$ is defined by

$$
\sigma^{\vee}=\left\{\xi \in V^{*} \mid \xi(v) \geq 0 \text { for all } v \in \sigma\right\} .
$$

There is an order reversing bijection $\tau \mapsto \tau^{\perp}$ between faces of $\sigma$ and faces of $\sigma^{\vee}$.

Let $X_{[\sigma]}$ denote the affine toric variety associated to the fan $[\sigma]$. The action of the complex torus $T=\operatorname{Hom}\left(V_{\mathbb{Z}}^{*}, \mathbb{C}^{*}\right)$ on $X_{[\sigma]}$ has one $T$-orbit $O_{\tau}$ for each face $\tau$ of $\sigma$, so that $O_{\rho} \subset \overline{O_{\tau}}$ if and only if $\tau \prec \rho$.

In $\$ 1.3$ below we define a full abelian subcategory $\mathcal{P}_{\Phi}=\mathcal{P}_{\Phi}\left(X_{[\sigma]}\right)$ of the category of perverse sheaves on $X_{[\sigma]}$. It depends on certain auxiliary data $\Phi$ attached to $[\sigma]$, which we call a "combinatorial completion", or "completion" for short. The simple objects of $\mathcal{P}_{\Phi}$ are $\left\{L_{\tau}\right\}_{\tau \in[\sigma]}$, where $L_{\tau}=I C \bullet\left(\overline{O_{\tau}} ; \mathbb{R}\right)$ is the intersection cohomology sheaf (with constant coefficients) supported on $\overline{O_{\tau}}$. The completion $\Phi$ gives restrictions on the allowed extensions between objects of $\mathcal{P}_{\Phi}$; one consequence is that $\mathcal{P}_{\Phi}$ has enough projectives and enough injectives.

A completion $\Phi$ of $[\sigma]$ induces a dual completion $\Phi^{\vee}$ of $\left[\sigma^{\vee}\right]$, so we get a category of perverse sheaves $\mathcal{P}_{\Phi^{\vee}}=\mathcal{P}_{\Phi^{\vee}}\left(X_{\left[\sigma^{\vee}\right]}\right)$ with simple objects $\left\{L_{\tau^{\perp}}\right\}_{\tau \in[\sigma]}$.

Let $I_{\tau} \in \mathcal{P}_{\Phi}$ and $I_{\tau^{\perp}} \in \mathcal{P}_{\Phi \vee}$ denote the injective hulls of $L_{\tau}, L_{\tau^{\perp}}$, so that $I=$ $\bigoplus_{\tau \prec \sigma} I_{\tau}$ and $I^{\vee}=\bigoplus_{\tau \prec \sigma} I_{\tau \perp}$ are injective generators of $\mathcal{P}_{\Phi}$ and $\mathcal{P}_{\Phi \vee}$, respectively. Also set $L=\bigoplus L_{\tau}, L^{\vee}=\bigoplus L_{\tau^{\perp}}$.

The first version of our main result is the following. It is exactly analogous to the main result of $\left[\underline{\mathrm{S}}\right.$, where the categories $\mathcal{P}_{\Phi}$ and $\mathcal{P}_{\Phi \vee}$ take the place of perverse sheaves on the flag variety.

1.1.1. Theorem. There is a canonical isomorphism of rings

$$
\operatorname{End}_{\mathcal{P}_{\Phi \vee}}\left(I^{\vee}\right)^{o p p} \cong \operatorname{Ext}_{\mathcal{P}_{\Phi}}^{\bullet}(L, L) .
$$

The ring on the right, with its natural grading, is Koszul.

Let us explain this result in more detail. For a graded ring $A$, let $A$-mod, $A$-Mod denote the categories of finitely generated graded and ungraded left $A$ modules, respectively. Let $A, A^{\vee}$ be the endomorphism rings of $I, I^{\vee}$, respectively, given gradings via Theorem 1.1.1. The functor $\operatorname{Hom}(-, I)$ gives an equivalence of categories $\mathcal{P}_{\Phi}^{o p p} \rightarrow A$-Mod, which sends $L$ to $A_{0}=A / A_{>0}$.

A graded ring $A=\bigoplus_{k \geq 0} A_{k}$ is called Koszul if (1) $A_{0}$ is semisimple, and (2) $\operatorname{Ext}_{A \text {-mod }}^{i}\left(A_{0}, A_{0}\langle-j\rangle\right)=0$ unless $i=j$, where $\langle j\rangle$ is the functor of shifting the grading down by $j$ (note that this is the opposite convention to the one in [BGS]).

The theorem can thus be interpreted as saying that $A^{\vee}$ is the opposite ring to Ext $_{A \text {-Mod }}\left(A_{0}, A_{0}\right)$. Rings related in this way are said to be Koszul dual to each other. 
1.2. Main result, second version. Theorem 1.1.1 can also be expressed by the existence of a duality functor between derived categories of $A$-modules and $A^{\vee}$ modules. Let $\widetilde{L}_{\tau}$ denote the simple object in $A$-mod concentrated in degree 0 whose ungraded version is $L_{\tau}$. Let $\widetilde{I}_{\tau}$ be its injective hull; it will be a graded version of $I_{\tau}$. Denote the simple and indecomposable injective objects of $A^{\vee}$-mod similarly.

1.2.1. Theorem. There is a contravariant equivalence of triangulated categories

$$
\kappa=\kappa_{\sigma}: D^{b}(A \text {-mod })^{o p p} \rightarrow D^{b}\left(A^{\vee} \text {-mod }\right),
$$

for which

(a) $\kappa(M[k])=(\kappa M)[-k]$ and $\kappa(M\langle k\rangle)=(\kappa M)[-k]\langle k\rangle$ for any $M \in D^{b}(A$-mod $)$, and

(b) $\kappa\left(\widetilde{L}_{\tau}\right) \cong \widetilde{I}_{\tau^{\perp}}$ and $\kappa\left(\widetilde{I}_{\tau}\right) \cong \widetilde{L}_{\tau^{\perp}}$ for any $\tau \prec \sigma$.

In [BGS] such a functor $\kappa$ is constructed for any pair $\left(A, A^{\vee}\right)$ of Koszul dual rings. We take the opposite point of view, however, first constructing $\kappa$ which satisfies Theorem 1.2.1, and then deducing Theorem 1.1.1 by the following argument.

Put $\widetilde{L}=\bigoplus \widetilde{L}_{\tau}$ and $\widetilde{I}^{\vee}=\bigoplus \widetilde{I}_{\tau^{\perp}}$. Then Theorem 1.2.1(b) says that $\kappa(\widetilde{L})=\widetilde{I}^{\vee}$. If we define $\operatorname{hom}^{\bullet}(M, N)=\bigoplus_{i, j \in \mathbb{Z}} \operatorname{Hom}(M, N[i]\langle j\rangle)$, then $\kappa$ induces a (non-gradingpreserving) ring isomorphism

$$
\operatorname{hom}_{D^{b}\left(A^{\vee} \text {-mod }\right)}^{\bullet}\left(\widetilde{I}^{\vee}, \widetilde{I}^{\vee}\right)^{o p p} \cong \operatorname{hom}_{D^{b}(A \text {-mod })}^{\bullet}(\widetilde{L}, \widetilde{L}) .
$$

Since higher Ext's between injective objects vanish, the left-hand side is just $A^{\vee}$; the isomorphism of Theorem 1.1.1 follows. Finally, for any $\tau, \rho \in[\sigma]$ we have

$$
\begin{aligned}
\operatorname{Ext}_{A-\text { mod }}^{i}\left(\widetilde{L}_{\tau}, \widetilde{L}_{\rho}\langle-j\rangle\right) & =\operatorname{Hom}_{D^{b}(A \text {-mod })}\left(\widetilde{L}_{\tau}, \widetilde{L}_{\rho}\langle-j\rangle[i]\right) \\
& =\operatorname{Hom}_{D^{b}\left(A^{\vee} \text {-mod }\right)}\left(\widetilde{I}_{\rho}^{\vee}, \widetilde{I}_{\tau}^{\vee}[i-j]\langle j\rangle\right) \\
& =\operatorname{Ext}_{A^{\vee} \text {-mod }}\left(\widetilde{I}_{\rho}^{\vee}, \widetilde{I}_{\tau}^{\vee}\langle j\rangle\right) .
\end{aligned}
$$

This vanishes if $i \neq j$, which proves that $A$ is Koszul.

1.3. Combinatorial completions. Our Koszul duality does not apply directly to the category of orbit-constructible perverse sheaves on toric varieties, because there are not enough injectives; there are local systems with arbitrarily long composition series on every orbit, so the same is true of perverse sheaves. We solve this problem by restricting to a subcategory of perverse sheaves with prescribed monodromy, in the following way.

Let $\Delta$ be a rational fan. More generally, we can let $\Delta$ be the set of cones of a fan which lie outside a subfan; we call such a set a "quasifan". A rational quasifan corresponds to a locally closed union of orbits in a toric variety, which we still denote $X_{\Delta}$.

For any cone $\sigma \in \Delta$, the $T$-orbit $O_{\sigma} \subset X_{\Delta}$ has a unique smallest $T$-invariant neighborhood $U_{\sigma}=\bigcup_{\tau \prec \sigma} O_{\tau}$. It is isomorphic to $O_{\sigma} \times X_{[\hat{\sigma}]}$, where $X_{[\hat{\sigma}]}$ is the affine toric variety corresponding to the cone $\hat{\sigma}$, which is equal to $\sigma$ as a set, but considered as a cone in $V_{\sigma}=\operatorname{span}(\sigma)$. This isomorphism is not canonical, however. Although there is a canonical projection $U_{\sigma} \rightarrow O_{\sigma}$, there is some freedom in choosing a projection $\pi_{\sigma}: U_{\sigma} \rightarrow X_{[\hat{\sigma}]}$.

Choosing the projection $\pi_{\sigma}$ is equivalent to fixing a subtorus of $T$ complementary to the stabilizer $T_{\sigma}$ of any point in $O_{\sigma}$. A combinatorial completion should be 
thought of as the choice of such a complement for every $\sigma$ in a compatible way. To put this in terms of fan geometry, we use the identification Lie $T \cong V \otimes_{\mathbb{R}} \mathbb{C}$, which takes Lie $T_{\sigma}$ to $V_{\sigma} \otimes_{\mathbb{R}} \mathbb{C}$.

1.3.1. Definition. A combinatorial completion $\Phi$ of a quasifan $\Delta$ is a choice of a subspace $\Phi_{\sigma} \subset V^{*}$ for every $\sigma \in \Delta$ so that

(1) if $\tau \prec \sigma$, then $\Phi_{\tau} \subset \Phi_{\sigma}$, and

(2) $V^{*}=\Phi_{\sigma} \oplus V_{\sigma}^{\perp}$ for every $\sigma \in \Delta$, where $V_{\sigma}^{\perp}$ denotes the annihilator of $V_{\sigma}$.

If, in addition, $\Delta$ is rational, and

(3) $\Phi_{\sigma} \cap V_{\mathbb{Z}}^{*}$ generates $\Phi_{\sigma}$ for every $\sigma \in \Delta$

(here $V_{\mathbb{Z}}^{*} \subset V^{*}$ is the lattice dual to $V_{\mathbb{Z}}$ ), then we say that $\Phi$ is rational.

The choice of $\Phi$ induces projection maps $\pi_{\sigma}: U_{\sigma} \rightarrow X_{[\hat{\sigma}]}$ for $\sigma \in \Sigma$; see $₫ 5.5$, Properties (1)-(3) are actually slightly weaker than the geometric idea we started with; they only imply that the resulting map $U_{\sigma} \rightarrow O_{\sigma} \times X_{[\hat{\sigma}]}$ is finite, rather than an isomorphism. However, this is sufficient for our purposes.

Now we can define the category $\mathcal{P}_{\Phi}\left(X_{\Delta}\right)$ referred to in 1.1 .

1.3.2. Definition. Let $\Phi$ be a rational combinatorial completion of a rational quasifan $\Delta$. Define $\mathcal{P}_{\Phi}\left(X_{\Delta}\right)$ to be the full subcategory of $\mathcal{P}\left(X_{\Delta}\right)$ consisting of objects $P$ so that for every $\sigma \in \Delta$ there exists an isomorphism $\left.P\right|_{U_{\sigma}} \cong \pi_{\sigma}^{*} P_{\sigma}[\operatorname{codim}(\sigma)]$, where $P_{\sigma}$ is a perverse sheaf on $X_{[\hat{\sigma}]}$. Such a $P$ will necessarily be constructible for the orbit stratification.

Remark. Another, perhaps less artificial, way to find a category of perverse sheaves with enough injectives is to take limits of perverse sheaves whose simple constituents are $L_{\tau}, \tau \in[\sigma]$. The resulting objects have unipotent monodromy on each orbit. They are not constructible in the classical sense, as the stalks may be infinite dimensional, but a satisfactory theory does exist. The category on the Koszul dual side is then (a mixed version of) the $T$-equivariant derived category. This is explained in $\mathrm{BL}$.

1.4. Combinatorial mixed sheaves. We construct our functor $\kappa$ by replacing the categories $D^{b}\left(A\right.$-mod) and $D^{b}\left(A^{\vee}\right.$-mod) by equivalent categories $\mathbf{D}_{\Phi}([\sigma])$ and $\mathbf{D}_{\Phi^{\vee}}\left(\left[\sigma^{\vee}\right]\right)$. These categories provide a combinatorial model for mixed sheaves on toric varieties, and should be of independent interest. Two other flavors of combinatorial mixed sheaves appear in $\mathrm{BL}$.

Given a fan or quasifan $\Delta$ with a completion $\Phi$ we define triangulated categories $\mathbf{D}(\Delta)$ and $\mathbf{D}_{\Phi}(\Delta)$; objects of these categories are complexes of sheaves on the finite topological space $\Delta$. The definition of these categories is valid even for nonrational fans, when the toric variety does not exist.

We also define the following additional structures relating these categories:

- a "forgetful functor" $F_{\Delta}: \mathbf{D}_{\Phi}(\Delta) \rightarrow \mathbf{D}(\Delta)$,

- pushforward and pullback functors $j^{*}, j^{!}, j_{*}, j_{\text {! }}$ defined for any inclusion $j$ of quasifans, and commuting with the forgetful functors,

- "twist" functors $\langle k\rangle, k \in \mathbb{Z}$, on both categories, commuting with $F_{\Delta}$, and

- $t$-structures on both categories, with corresponding abelian subcategories of perverse objects $\mathbf{P}_{\Phi}(\Delta)$ and $\mathbf{P}(\Delta)$.

$F_{\Delta}$ is compatible with these $t$-structures, in the sense that the $t$-structure on $\mathbf{D}_{\Phi}(\Delta)$ is pulled back from the $t$-structure on $\mathbf{D}(\Delta)$. In particular we have $\mathbf{P}_{\Phi}(\Delta)=$ 
$F_{\Delta}^{-1}(\mathbf{P}(\Delta))$. The twist functors $\langle k\rangle$ are also $t$-exact, and so they induce functors on $\mathbf{P}_{\Phi}(\Delta)$ and $\mathbf{P}(\Delta)$.

Up to isomorphism and twists, there is a unique simple object $L_{\tau}^{\bullet}$ in $\mathbf{P}_{\Phi}(\Delta)$ which is supported on the closure of $\tau$. $F_{\Delta}$ induces a bijection between the isomorphism classes of simple objects of $\mathbf{P}_{\Phi}(\Delta)$ and of $\mathbf{P}(\Delta)$.

We also show that $F_{\Delta}$ embeds $\mathbf{P}_{\Phi}(\Delta)$ as a full subcategory of $\mathbf{P}(\Delta)$.

1.4.1. Proposition. $\mathbf{P}_{\Phi}(\Delta)$ has enough injectives, and we have an equivalence of categories $D^{b}\left(\mathbf{P}_{\Phi}(\Delta)\right) \cong \mathbf{D}_{\Phi}(\Delta)$.

If the (quasi)fan $\Delta$ is rational and $X_{\Delta}$ is the corresponding toric variety, we define in $\S 5$ a topological realization functor

$$
\operatorname{real}_{\Delta}: \mathbf{D}(\Delta) \rightarrow D_{u}^{b}\left(X_{\Delta}\right)
$$

where $D_{u}^{b}\left(X_{\Delta}\right)$ is the subcategory $D^{b}\left(X_{\Delta}\right)$ consisting of objects whose cohomology sheaves restricted to any orbit are local systems with unipotent monodromy. We conjecture that this can be factored through a "mixed" realization functor sending $\mathbf{D}(\Delta)$ to mixed Hodge modules or mixed $l$-adic sheaves on $X_{\Delta}$.

1.4.2. Theorem. The realization functor real $_{\Delta}$ satisfies:

(1) For each $k \in \mathbb{Z}$, there is a natural isomorphism

$$
\operatorname{real}_{\Delta} \circ\langle k\rangle \simeq \text { real }_{\Delta} \text {. }
$$

(2) For any objects $S_{1}, S_{2}^{\bullet} \in \mathbf{D}(\Delta)$, the induced map

$$
\bigoplus_{k \in \mathbb{Z}} \operatorname{Hom}_{\mathbf{D}(\Delta)}\left(S_{1}^{\bullet}, S_{2}^{\bullet}\langle k\rangle\right) \rightarrow \operatorname{Hom}_{D_{u}^{b}\left(X_{\Delta}\right)}\left(\operatorname{real}_{\Delta} S_{1}^{\bullet}, \operatorname{real}_{\Delta} S_{2}^{\bullet}\right)
$$

is an isomorphism.

(3) If $j: \Sigma \rightarrow \Delta$ is an inclusion of quasifans, there exist natural isomorphisms $j^{*} \circ$ real $_{\Delta} \simeq \operatorname{real}_{\Sigma} \circ j^{*}, R j_{*} \circ \mathrm{real}_{\Sigma} \simeq \mathrm{real}_{\Delta} \circ j_{*}$, and similarly for $j^{!}, j_{!}$. Here we use the same letter $j$ to denote the inclusion $X_{\Sigma} \rightarrow X_{\Delta}$.

(4) An object $S^{\bullet} \in \mathbf{D}(\Delta)$ is in $\mathbf{P}(\Delta)$ if and only if real $S_{\Delta}$ is a perverse sheaf.

(5) $\left.\operatorname{real}_{\Delta}\right|_{\mathbf{P}(\Delta)}$ sends simples to simples.

If $\Delta$ is a rational quasifan with rational completion $\Phi$, let real ${ }_{\Delta, \Phi}$ denote the composition

$$
\mathbf{D}_{\Phi}(\Delta) \rightarrow \mathbf{D}(\Delta) \stackrel{\text { real }_{\Delta}}{\longrightarrow} D_{u}^{b}\left(X_{\Delta}\right)
$$

it restricts to an exact functor $\mathbf{P}_{\Phi}(\Delta) \rightarrow \mathcal{P}\left(X_{\Delta}\right)$.

\subsubsection{Theorem.}

(1) If $S^{\bullet} \in \mathbf{P}(\Delta)$, then $S^{\bullet}$ is in $\mathbf{P}_{\Phi}(\Delta)$ if and only if $\operatorname{real}_{\Delta}\left(S^{\bullet}\right)$ is in $\mathcal{P}_{\Phi}(\Delta)$.

(2) real $_{\Delta, \Phi}$ takes injective objects of $\mathbf{P}_{\Phi}(\Delta)$ to injective objects in $\mathcal{P}_{\Phi}(\Delta)$, and all injectives in $\mathcal{P}_{\Phi}(\Delta)$ can be obtained in this way.

(3) For any two objects $S_{1}^{\bullet}, S_{2}^{\bullet} \in \mathbf{P}_{\Phi}(\Delta)$, and any $i \geq 0$, we have

$$
\operatorname{Ext}_{\mathcal{P}_{\Phi}}^{i}\left(\operatorname{real}_{\Delta, \Phi} S_{1}^{\bullet}, \operatorname{real}_{\Delta, \Phi} S_{2}^{\bullet}\right) \cong \bigoplus_{k \in \mathbb{Z}} \operatorname{Ext}_{\mathbf{P}_{\Phi}(\Delta)}^{i}\left(S_{1}^{\bullet}, S_{2}^{\bullet}\langle k\rangle\right) .
$$

Let us explain the intuition behind the construction of $\mathbf{D}(\Delta)$. There is a "combinatorial moment map" $\mu: X_{\Delta} \rightarrow \Delta$ which sends all points of $O_{\sigma} \subset X_{\Delta}$ to $\sigma$; put another way, it is the quotient map $X_{\Delta} \rightarrow X_{\Delta} / T$. Then $\Lambda=\bigoplus_{j} R^{j} \mu_{*} \mathbb{R}_{X_{\Delta}}$ is a sheaf of graded rings on $\Delta$ whose stalk $\Lambda_{\sigma}$ at a face $\sigma$ is the cohomology ring $H^{\bullet}\left(O_{\sigma} ; \mathbb{R}\right)$, which is an exterior algebra. 
The cohomology of an object $S \in D^{b}\left(X_{\Delta}\right)$ is naturally a $\Lambda$-module. More information is preserved if we take the derived push-forward; $R \mu_{*} S$ becomes an object in the category of $d g$-modules over $\Lambda$, considered as a sheaf of differential graded algebras with trivial differential. This works because the torus $T$ is formal, meaning that the $d g$-algebra $\Omega \bullet(T)$ of differential forms is quasi-isomorphic to its own cohomology. A similar idea appears in Lunts [L].

Mixed complexes are "bigraded" objects: there is one shift operation on degrees, and another on weights. Accordingly, objects of our category $\mathbf{D}(\Delta)$ are complexes of graded $\Lambda$-modules rather than $d g$-modules. This gives two shift operators, one shifting the complex degree and another shifting the grading. The $t$-exact twist functor $\langle k\rangle$ of Theorem 1.4.2 is a diagonal combination of both types of shift.

Remark. A key reason we can describe weights so simply is that for each $i$ the cohomology $H^{i}\left(O_{\tau} ; \mathbb{R}\right)$ is pure (of weight $2 i$ ). To make a similar construction on flag varieties, one wants to replace the exterior algebras $\Lambda_{\sigma}$ by the stalk cohomology groups of $j_{S *} \mathbb{R}_{S}$, where $S$ is a Bruhat cell and $j_{S}$ its inclusion into the flag variety. Unfortunately this cohomology is not pure, as follows from an example of Boe ([Boe, see also [SSV]). We hope that it will still be possible to understand Koszul duality on flag varieties using these techniques, perhaps by staying on the level of $d g$-algebras.

1.5. Main result, third version. If $\Delta=[\sigma]$ for a rational fan $\sigma$, the results explained in the last section imply $\mathbf{D}_{\Phi}([\sigma]) \cong D^{b}\left(\mathbf{P}_{\Phi}([\sigma])\right) \cong D^{b}(A$-mod), where $A$ is the endomorphism ring of an injective generator of $\mathcal{P}_{\Phi}\left(X_{\Delta}\right)$, and the grading on $A$ is given by lifting an injective generator of $\mathcal{P}_{\Phi}\left(X_{[\sigma]}\right)$ to $\mathbf{P}_{\Phi}([\sigma])$, taking the endomorphism ring, and using Theorem 1.4.3(3).

Thus to prove Theorem 1.2.1 and hence Theorem 1.1.1 it is enough to construct a functor

$$
\kappa: \mathbf{D}_{\Phi}([\sigma])^{o p p} \rightarrow \mathbf{D}_{\Phi \vee}\left(\left[\sigma^{\vee}\right]\right)
$$

satisfying the properties of Theorem 1.2.1 The grading shift on $A$-modules becomes the twist $\langle k\rangle$, and simples and injective modules in $A$-mod are replaced by simple and injective objects of $\mathbf{P}_{\Phi}([\sigma])$. Note that we only fixed these simples up to a twist $\langle k\rangle$, but property (a) of Theorem 1.2.1 implies that there will be only one choice of lift for which $\kappa\left(L_{\tau}^{\bullet}\right)$ is perverse.

1.6. Duality between costandard objects. To construct the functor $\kappa$ and to prove Theorem 4.2.1 we look first at costandard objects, which have simpler topology than either injective or simple objects. If $\tau \prec \sigma$, the costandard object $N_{\tau}^{\bullet} \in \mathbf{P}_{\Phi}([\sigma])$ is the (derived) "lower star" extension of the rank one constant local system $\mathbb{R}_{O_{\tau}}$ to $X_{[\sigma]}$. It is a well-known theme in representation theory that costandards lie midway between simples and injectives, so it is not surprising that $\kappa$ will send costandards to costandards. In fact, requiring this dictates our definition of $\kappa$, since costandards generate the category $\mathbf{D}_{\Phi}(\Delta)$.

In this way we get a functor which is clearly an equivalence of categories, which has the shift properties of part (a) of Theorem 1.2.1, and which satisfies $\kappa\left(N_{\tau}^{\bullet}\right) \cong N_{\tau^{\perp}}$ for all $\tau \in[\sigma]$. It remains to show that $\kappa$ interchanges simples and injectives. We do this by showing that the usual "extend-truncate-repeat" procedure for constructing the simple object $L_{\tau}^{\cdot}$, becomes under $\kappa$ a construction of $I_{\tau^{\perp}}$. 
Roughly, the construction of $I_{\tau^{\perp}}$ starts with $N_{\tau^{\perp}}$ and extends by $N_{\rho}^{\bullet}$ for successively smaller $\rho$ (larger strata!), killing off any possible Ext ${ }^{1} \mathrm{~s}$ that remain at each stage.

This follows from the purity of the stalks of $L_{\dot{\tau}}$, which in our combinatorial language says that the stalk cohomology as a bigraded vector space lies all on a diagonal. We deduce purity from the Hard Lefschetz theorem for toric varieties, which was recently proved for nonrational fans by Karu $\mathrm{Ka}$, using the theory of equivariant combinatorial sheaves on fans of $[\mathrm{BrL}, \mathrm{BBFK}]$. To translate this result to our category $\mathbf{D}(\Delta)$ we use the classical Koszul duality between symmetric and exterior algebras, which relates equivariant and ordinary cohomology and sheaf theory GKM].

1.7. Outline of the paper. We give a brief overview of the sections of this paper. Section 2 collects a few preliminary definitions regarding gradings, cones and fans, and sheaves on fans. Section 3 is concerned with the definition and basic properties of the categories $\mathbf{D}(\Delta)$ and $\mathbf{D}_{\Phi}(\Delta)$. The categories are defined in $\S \$ 3.1$ and 3.2 , The pushforward and pullback functors are described in $\$ 3.3$ and the $t$-structure on these categories is defined in $\$ 3.4$. The injective and costandard objects in the core of this $t$-structure are studied in $\$ \$ 3.6$ and 3.7. In $\$ 3.8$ we work out in detail the structure of these categories in the simplest nontrivial case of a single one-dimensional cone.

Section 4 defines the Koszul functor $\kappa$ (4.2), proves the purity of the stalks and costalks of the intersection cohomology objects (\$4.3), and proves that $\kappa$ interchanges simples and injectives (4.4).

In Section 5, we define the functor real $\Delta$, and show how the combinatorial functors, completions, $t$-structures, etc., correspond to topological properties of sheaves. In particular, this section contains proofs of Theorems 1.4.2 and 1.4.3.

\section{Preliminaries}

2.1. Gradings, shifts, and total Hom. We begin by fixing notation regarding gradings.

Let $A=\bigoplus_{i} A_{i}$ be a finitely generated graded $\mathbb{R}$-algebra, or more generally a sheaf of such algebras on a finite topological space. Let $A$-Mod and $A$-mod denote the categories of finitely generated $A$-modules and finitely generated graded $A$ modules, respectively. Let $\{1\}: A$-mod $\rightarrow A$-mod be the shift of grading given by $(M\{1\})_{i}=M_{i+1}$. Let $\{n\}$ be the $n$-fold composition of $\{1\}$, or the $-n$-fold composition of its inverse if $n<0$.

For objects $M, N \in A$-mod, we put $\operatorname{Hom}_{i}(M, N)=\operatorname{Hom}_{A-\bmod }(M, N\{i\})$, and set

$$
\operatorname{hom}(M, N)=\bigoplus_{i \in \mathbb{Z}} \operatorname{Hom}_{i}(M, N) .
$$

If we assume that $\operatorname{dim}_{\mathbb{R}} A<\infty$, then $\operatorname{hom}(M, N)$ is finite dimensional, so it can be considered as an object in $\mathbb{R}$-mod with $\operatorname{Hom}_{i}(M, N)$ placed in degree $i$.

Suppose now that $\mathcal{D}$ is a full triangulated subcategory of either the homotopy category $K(A$-mod $)$ or its derived category $D(A$-mod), and that $\mathcal{D}\{j\}=\mathcal{D}$ for any $j \in \mathbb{Z}$. If $M^{\bullet}$ is a complex in $\mathcal{D}$, we say that elements of $M_{j}^{i}$ are in bidegree $(i, j)$. 
Given complexes $M^{\bullet}, N^{\bullet} \in \mathcal{D}$, we define

$$
\begin{aligned}
\operatorname{Hom}_{j}^{i}\left(M^{\bullet}, N^{\bullet}\right) & =\operatorname{Hom}_{\mathcal{D}}\left(M^{\bullet}, N^{\bullet}[i]\{j\}\right), \\
\operatorname{hom}^{\bullet}\left(M^{\bullet}, N^{\bullet}\right) & =\bigoplus_{i, j \in \mathbb{Z}} \operatorname{Hom}_{j}^{i}\left(M^{\bullet}, N^{\bullet}\right) .
\end{aligned}
$$

We consider $\operatorname{hom}^{\bullet}\left(M^{\bullet}, N^{\bullet}\right)$ as an object in $D^{b}(\mathbb{R}$-mod) with trivial differential.

We have a tensor product functor $D^{b}(\mathbb{R}-$ mod $) \times \mathcal{D} \rightarrow \mathcal{D}$ given by

$$
V \otimes M^{\bullet}=\bigoplus_{i, j \in \mathbb{Z}} H^{i}(V)_{j} \otimes M^{\bullet}[-i]\{-j\} .
$$

For any $M^{\bullet}, N^{\bullet} \in \mathcal{D}$, there is a natural morphism

$$
\operatorname{hom}^{\bullet}\left(M^{\bullet}, N^{\bullet}\right) \otimes M^{\bullet} \rightarrow N^{\bullet} .
$$

For formal reasons it will be useful to consider complexes $\left(M^{\bullet}, d\right)$, where both the complex and the degree are allowed to be half-integers. We let $\Xi$ be the lattice $\left\{(i, j) \in \mathbb{R}^{2} \mid i+j, i-j \in \mathbb{Z}\right\}$, and we let $K_{h}(A$-mod $), D_{h}(A$-mod) be the homotopy category and derived category of the category of complexes $M^{\bullet}=\bigoplus_{(i, j) \in \Xi} M_{j}^{i}$ with differential $d: M_{j}^{i} \rightarrow M_{j}^{i+1}$ and an action of $A$ so that $A_{k} M_{j}^{i} \subset M_{j+k}^{i}$. All the definitions made above go over to this setting in an obvious way.

2.2. Cones. We fix some notations concerning cones and fans. A cone in a finitedimensional real vector space $V$ is a subset of the form $\sigma=\mathbb{R}_{\geq 0} v_{1}+\cdots+\mathbb{R}_{\geq 0} v_{k}$, $v_{1}, \ldots, v_{k} \in V$, which contains no line through 0 . We include the possibility that $\sigma$ is the zero cone $\{0\}$. The dimension $\operatorname{dim} \sigma$ of a cone $\sigma$ is the dimension of the vector space $V_{\sigma}=\operatorname{span}(\sigma)$.

If a dual vector $\xi \in V^{*}$ satisfies $\sigma \subset \xi^{-1}\left(\mathbb{R}_{\geq 0}\right)$, we call the set $\tau=\sigma \cap \xi^{-1}(0)$ a face of $\sigma$. It is again a cone in $V$, and we write $\tau \prec \sigma$ to indicate the relation that $\tau$ is a face of $\sigma$. If $[\sigma]$ denotes the set of all faces of $\sigma$, then $([\sigma], \prec)$ is a finite ranked lattice, with maximal element $\sigma$ and minimal element $o=\{0\}$.

Remark. Note that several of our constructions, including the definition of toric varieties, involve taking the dual cone $\sigma^{\vee}$ to $\sigma$ or the annihilator $V_{\sigma}^{\perp} \subset V^{*}$ of the space $V_{\sigma}$. These notions obviously depend on the ambient space $V$, and we consider the choice of ambient vector space to be a part of the definition of a cone.

2.3. Fans. A fan $\Delta$ in $V$ is a finite collection of cones in $V$ for which

(1) $\sigma \cap \tau$ is a face of both $\sigma$ and $\tau$ whenever $\sigma$ and $\tau$ are in $\Delta$, and

(2) for each $\sigma \in \Delta$, we have $[\sigma] \subset \Delta$.

A quasifan is a finite collection of cones $\Delta$ satisfying (1) and

$\left(2^{\prime}\right)$ if $\sigma, \tau \in \Delta$, then $\tau \prec \rho \prec \sigma$ implies $\rho \in \Delta$.

Clearly every fan is a quasifan. A quasifan $\Delta$ has a unique smallest fan containing it, namely $[\Delta]=\bigcup_{\sigma \in \Delta}[\sigma]$.

Given a quasifan $\Delta$, we give it the topology generated by basic open sets $[\sigma] \cap \Delta$, $\sigma \in \Delta$. Quasifans $\Sigma \subset \Delta$ are just the locally closed subsets.

Suppose that $V$ contains a lattice $V_{\mathbb{Z}}$ for which $V=V_{\mathbb{Z}} \otimes_{\mathbb{Z}} \mathbb{R}$. A subspace $W \subset V$ is called rational if $W \cap V_{\mathbb{Z}}$ spans $W$. A quasifan $\Delta$ is said to be rational if $V_{\sigma}$ is rational for every $\sigma \in \Delta$; we call a cone $\sigma$ rational if $[\sigma]$ is rational. 
2.4. Sheaves on fans. Let $\Delta$ be a quasifan in $V$, with the topology described above.

2.4.1. Proposition. There is an equivalence of categories between the category of sheaves of abelian groups on $\Delta$ and the category of data $\left(\left\{S_{\sigma}\right\},\left\{r_{\tau, \sigma}\right\}\right)$, where

- $S_{\sigma}$ is an abelian group for all $\sigma \in \Delta$,

- for every pair of faces $\tau \prec \sigma$ in $\Delta, r_{\tau, \sigma}: S_{\sigma} \rightarrow S_{\tau}$ is a homomorphism, and

- whenever $\rho \prec \tau \prec \sigma$, we have $r_{\rho, \tau} r_{\tau, \sigma}=r_{\rho, \sigma}$.

A morphism $\phi:\left(\left\{S_{\sigma}\right\},\left\{r_{\tau, \sigma}\right\}\right) \rightarrow\left(\left\{S_{\sigma}^{\prime}\right\},\left\{r_{\tau, \sigma}^{\prime}\right\}\right)$ in this category is a collection of maps $\phi_{\sigma}: S_{\sigma} \rightarrow S_{\sigma}^{\prime}$ for every $\sigma \in \Delta$ satisfying $\phi_{\tau} r_{\tau, \sigma}=r_{\tau, \sigma}^{\prime} \phi_{\sigma}$ for every $\tau \prec \sigma$.

Proof. Given a sheaf $S$, let $S_{\sigma}=\Gamma(S ;[\sigma] \cap \Delta)$; this is the stalk of $S$ at $\sigma$, since $[\sigma] \cap \Delta$ is the smallest open set containing $\sigma$. The homomorphisms $r_{\tau, \sigma}$ are then given by restriction of sections $\Gamma(S ;[\sigma] \cap \Delta) \rightarrow \Gamma(S ;[\tau] \cap \Delta)$.

The inverse functor is also easy to describe: given data $\left(\left\{S_{\sigma}\right\},\left\{r_{\tau, \sigma}\right\}\right)$ and an open set $U \subset \Delta$, the space of sections $\Gamma(S ; U)$ is the inverse $\operatorname{limit}_{\leftarrow} \lim _{\sigma \in U} S_{\sigma}$.

We will pass freely between these points of view on sheaves without comment.

The same statement holds for sheaves of rings, replacing "abelian group" with "ring" everywhere. Furthermore, given a sheaf $\mathcal{R}$ of rings on $\Sigma$, we can describe the category $\mathcal{R}$-mod of sheaves of $\mathcal{R}$-modules as the category of pairs $\left(\left\{S_{\sigma}\right\},\left\{r_{\tau, \sigma}\right\}\right)$, where each $S_{\sigma}$ is an $\mathcal{R}_{\sigma}$-module and each $r_{\tau, \sigma}: S_{\sigma} \rightarrow S_{\tau}$ is a homomorphism of $\mathcal{R}_{\sigma}$-modules. Here $S_{\tau}$ becomes an $\mathcal{R}_{\sigma}$-module via $\mathcal{R}_{\sigma} \rightarrow \mathcal{R}_{\tau}$.

We also need to describe pullback and pushforward functors in this language. Let $\Sigma$ be a sub-quasifan of $\Delta$, with inclusion $j$. If $\mathcal{R}=\mathcal{R}_{\Delta}$ is a sheaf of rings on $\Delta$, the restriction $\mathcal{R}_{\Sigma}=\left.\mathcal{R}_{\Delta}\right|_{\Sigma}$ has the same stalks and restriction maps, but only for $\sigma, \tau \in \Sigma$. In the same way, given an object $S \in \mathcal{R}_{\Delta}$-mod, the pull-back $j^{*} S$ is given by keeping only the stalks $S_{\sigma}$ for $\sigma \in \Sigma$.

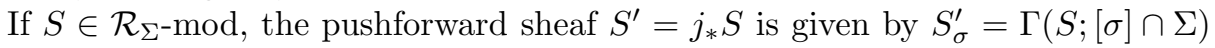
for $\sigma \in \Delta$. This is an $\mathcal{R}_{\sigma}$-module, since it is the inverse limit of $S_{\tau}$ for $\tau \in[\sigma] \cap \Sigma$, each of which is an $\mathcal{R}_{\sigma}$-module by the homomorphism $\mathcal{R}_{\sigma} \rightarrow \mathcal{R}_{\tau}$.

Finally, we define an "extension by zero" functor $j$ !! in this language (we use this nonstandard notation to avoid confusion with its derived version, defined in $\$ 3.3$ ). For $S \in \mathcal{R}_{\Sigma}$-mod, we let $\left(j_{! !} S\right)_{\tau}=S_{\tau}$ if $\tau \in \Sigma$, and $\left(j_{! !} S\right)_{\tau}=0$ otherwise.

\section{Combinatorial Sheaves ON FAnS}

3.1. The basic definition. Given a quasifan $\Delta$ in $V$, define a sheaf of graded rings $\Lambda=\Lambda_{\Delta}$ on it as follows. Let the stalk $\Lambda_{\sigma}$ be the exterior algebra $\Lambda\left(V_{\sigma}^{\perp}\right)$, with the standard grading where $\Lambda^{1}\left(V_{\sigma}^{\perp}\right)=V_{\sigma}^{\perp}$ has degree 1 . If $\tau \prec \sigma$ are cones in $\Delta$, let $\Lambda_{\sigma} \rightarrow \Lambda_{\tau}$ be the natural homomorphism induced by the inclusion $V_{\sigma}^{\perp} \rightarrow V_{\tau}^{\perp}$.

Let $\Lambda_{\Delta}$-mod denote the abelian category of finitely generated $\Lambda_{\Delta}$-modules. We write $A \mapsto A\{k\}$ for the automorphism of $\Lambda_{\Delta}$-mod which shifts the grading down by $k \in \mathbb{Z}$.

For a cone $\sigma \in \Sigma$, let $i_{\sigma}:\{\sigma\} \rightarrow \Sigma$ denote the inclusion, and define $J_{\sigma}=i_{\sigma *} \Lambda_{\sigma}$. Using Proposition 2.4.1, $J_{\sigma}$ has a simple description: if $\sigma \prec \tau$, then $\left(J_{\sigma}\right)_{\tau}=\Lambda_{\sigma}$ with the induced action of $\Lambda_{\tau}$, while $\left(J_{\sigma}\right)_{\tau}=0$ if $\sigma \nprec \tau$. The restriction map $r_{\tau, \rho}$ is the identity whenever $\sigma \prec \tau \prec \rho$. 
3.1.1. Proposition. The category $\Lambda_{\Delta}$-mod has enough injectives. An object in $\Lambda_{\Delta}$-mod is injective if and only if it is injective as a sheaf of $\mathbb{R}$-modules. $J_{\sigma}$ is injective for every $\sigma \in \Delta$, and any injective object is a direct sum of $J_{\sigma}\{k\}$ for various $\sigma \in \Delta, k \in \mathbb{Z}$.

Proof. The injectivity of $J_{\sigma}$ follows from the adjunction between $i_{\sigma}^{*}$ and $i_{\sigma *}$. For any $S \in \Lambda_{\Delta}$-mod, the natural homomorphism

$$
S \rightarrow \bigoplus_{\sigma \in \Delta} i_{\sigma *} i_{\sigma}^{*} S
$$

is injective, so $S$ embeds into the injective object $\bigoplus_{\sigma \in \Delta} i_{\sigma *} I_{\sigma}$, where for each $\sigma$ the $\Lambda_{\sigma}$-module $I_{\sigma}$ is an injective hull of $i_{\sigma}^{*} S$.

Let $I n j_{\Delta}$ be the full subcategory of injectives in $\Lambda_{\Delta}$-mod. We define a triangulated category $\mathbf{D}(\Delta)$ to be the homotopy category $K_{h}^{b}\left(\operatorname{Inj}_{\Delta}\right)$ of bounded complexes of objects in $I n j_{\Delta}$, with the "half grading" described in $\$ 2.1$. This is equivalent to the full subcategory of $D_{h}^{b}\left(\Lambda_{\Delta}\right.$-mod) of objects whose cohomology sheaves are locally free, where $S \in \Lambda_{\Delta}$-mod is called locally free if each stalk $S_{\sigma}$ is a free $\Lambda_{\sigma}$-module.

Define a functor $\Gamma_{\sigma}: \mathbf{D}(\sigma) \rightarrow D_{h}^{b}(\mathbb{R}$-mod) by

$$
\Gamma_{\sigma}\left(S^{\bullet}\right)_{q}^{p}=q \text { th graded piece of } H^{p}\left(S^{\bullet} \otimes_{\Lambda_{\sigma}} \mathbb{R}\right),
$$

with trivial differential. In terms of our dictionary with sheaves on the toric variety $X_{\Delta}, \Gamma_{\sigma}\left(S^{\bullet}\right)$ should be thought of as the stalk of $S^{\bullet}$ at a point of $O_{\sigma}$.

3.2. Combinatorial completions and stable sheaves. Let $\Delta$ be a fan in the vector space $V$. Recall from $\$ 1.3$ that a combinatorial completion $\Phi$ of $\Delta$ is a choice for each $\tau \in \Delta$ of a complement $\Phi_{\tau}$ to $V_{\tau}^{\perp}$ in $V^{*}$ satisfying $\Phi_{\rho} \subset \Phi_{\tau}$ whenever $\rho \prec \tau$. Such completions always exist. We can, for instance, choose a nondegenerate bilinear form on $V^{*}$ and take $\Phi_{\tau}$ to be the orthogonal complement of $V_{\tau}^{\perp}$.

Fix a completion $\Phi$ of $\Delta$. Given faces $\tau \prec \sigma$ of $\Delta$, we define $\Phi_{\sigma}^{\tau}=V_{\tau}^{\perp} \cap \Phi_{\sigma}$. It is a complement to $V_{\sigma}^{\perp}$ in $V_{\tau}^{\perp}$. Easy linear algebra shows that for any cones $\rho \prec \tau \prec \sigma$, we have

$$
\Phi_{\sigma}^{\rho}=\Phi_{\sigma}^{\tau} \oplus \Phi_{\tau}^{\rho} .
$$

3.2.2. Definition. The category $\Lambda_{\Delta}-\bmod _{\Phi}$ of $\Phi$-stable sheaves has as objects the pairs $\left(S,\left\{S_{\sigma}^{\natural}\right\}_{\sigma \in \Delta}\right)$, where $S \in \Lambda_{\Delta}$-mod and the stabilization $\left\{S_{\sigma}^{\natural}\right\}$ is a choice for each $\sigma \in \Delta$ of a graded vector subspace of $S_{\sigma}^{\natural} \subset S_{\sigma}$ for which:

(1) For each $\sigma \in \Delta$, multiplication gives an isomorphism

$$
\Lambda_{\sigma} \otimes S_{\sigma}^{\natural} \rightarrow S_{\sigma}
$$

(in particular, $S_{\sigma}$ is a free $\Lambda_{\sigma}$-module), and

(2) if $\tau \prec \sigma$ are faces in $\Delta$, then

$$
r_{\tau, \sigma}\left(S_{\sigma}^{\natural}\right) \subset \Lambda\left(\Phi_{\sigma}^{\tau}\right) \cdot S_{\tau}^{\natural} .
$$

A morphism $\phi:\left(R,\left\{R_{\sigma}^{\natural}\right\}\right) \rightarrow\left(S,\left\{S_{\sigma}^{\natural}\right\}\right)$ is a morphism $\phi: R \rightarrow S$ for which $\phi\left(R_{\sigma}^{\natural}\right) \subset$ $S_{\sigma}^{\natural}$ for all $\sigma \in \Delta$.

Forgetting the stabilization gives a functor $\Lambda_{\Delta}-\bmod _{\Phi} \rightarrow \Lambda_{\Delta}$-mod. 
3.2.3. Proposition. The category $\Lambda_{\Delta}-\bmod _{\Phi}$ is abelian, and has enough injectives. The indecomposable injective $J_{\tau}$ in $\Lambda_{\Delta}$-mod has a unique stabilization given by $\left(J_{\tau}\right)_{\sigma}^{\natural}=\Lambda\left(\Phi_{\sigma}^{\tau}\right)$ if $\tau \prec \sigma$, and 0 otherwise. The resulting objects are, up to isomorphism and shifts of grading, the only indecomposable injectives.

Most of the time we will simply write $S$ instead of $\left(S,\left\{S_{\sigma}^{\natural}\right\}\right)$ when discussing objects of $\Lambda_{\Delta}-\bmod _{\Phi}$. In particular we will use the same symbol $J_{\tau}$ to denote an injective object in $\Lambda_{\Delta}-\bmod _{\Phi}$ and in $\Lambda_{\Delta}$-mod.

Let $\operatorname{Inj}_{\Delta, \Phi}$ be the full subcategory of injectives in $\Lambda_{\Delta}-\bmod _{\Phi}$, and define the triangulated category $\mathbf{D}_{\Phi}(\Delta)$ to be the homotopy category $K_{h}^{b}\left(\operatorname{Inj}_{\Delta, \Phi}\right)$. Forgetting the stabilizations produces a functor $F_{\Delta}: \mathbf{D}_{\Phi}(\Delta) \rightarrow \mathbf{D}(\Delta)$.

Intuitively, by passing to the category $\mathbf{D}_{\Phi}(\Delta)$ we have made the "strata" $\sigma \in \Delta$ contractible. The following lemma makes this precise.

3.2.4. Lemma. The composed functor

$$
\mathbf{D}_{\Phi}(\sigma) \stackrel{F_{\sigma}}{\longrightarrow} \mathbf{D}(\sigma) \stackrel{\Gamma_{\sigma}}{\longrightarrow} D_{h}^{b}(\mathbb{R}-\mathrm{mod})
$$

is an equivalence of categories.

A more precise description of the structure of the category $I n j_{\Delta, \Phi}$ will be useful later. Let hom ${ }_{\Delta, \Phi}=\operatorname{hom}_{I n j_{\Delta, \Phi}}$ in what follows.

3.2.5. Proposition. For any faces $\sigma, \tau \in \Delta$ there is a canonical identification

$$
\operatorname{hom}_{\Delta, \Phi}\left(J_{\tau}, J_{\sigma}\right)=\left\{\begin{array}{cl}
\Lambda\left(\Phi_{\sigma}^{\tau}\right)^{*} & \text { if } \tau \prec \sigma, \\
0 & \text { otherwise }
\end{array}\right.
$$

as graded vector spaces. If $\rho \prec \tau \prec \sigma$, then under the identification (3.2.6), the composition map

$$
\operatorname{hom}_{\Delta, \Phi}\left(J_{\tau}, J_{\sigma}\right) \otimes \operatorname{hom}_{\Delta, \Phi}\left(J_{\rho}, J_{\tau}\right) \rightarrow \operatorname{hom}_{\Delta, \Phi}\left(J_{\rho}, J_{\sigma}\right)
$$

is the inverse transpose of the wedge product $\Lambda\left(\Phi_{\sigma}^{\tau}\right) \otimes \Lambda\left(\Phi_{\tau}^{\rho}\right) \rightarrow \Lambda\left(\Phi_{\sigma}^{\rho}\right)$.

Note that (3.2.1) shows the wedge product above is an isomorphism. In (3.2.6) the grading is given by $\Lambda\left(\Phi_{\sigma}^{\tau}\right)^{*}=\operatorname{hom}_{\mathbb{R}-\bmod }\left(\Lambda\left(\Phi_{\sigma}^{\tau}\right), \mathbb{R}\right)$. In other words, we let elements of $\left(\Phi_{\sigma}^{\tau}\right)^{*}$ live in degree -1 .

Proof. The identification (3.2.6) is given by

$$
\begin{aligned}
\operatorname{hom}_{\Delta, \Phi}\left(J_{\tau}, J_{\sigma}\right) \rightarrow \operatorname{hom}_{\sigma, \Phi}\left(i_{\sigma}^{*} J_{\tau}, i_{\sigma}^{*} J_{\sigma}\right) & \rightarrow \operatorname{hom}_{\mathbb{R}-\bmod }\left(\left(J_{\tau}\right)_{\sigma}^{\natural},\left(J_{\sigma}\right)_{\sigma}^{\natural}\right) \\
& =\operatorname{hom}_{\mathbb{R}-\bmod }\left(\Lambda\left(\Phi_{\sigma}^{\tau}\right), \mathbb{R}\right) .
\end{aligned}
$$

Checking that this is an isomorphism and identifying the composition homomorphism is easy.

3.3. Pushforward and pullback functors. Fix a quasifan $\Delta$ and a sub-quasifan $\Sigma$; let $i: \Sigma \rightarrow \Delta$ be the inclusion. Let $\Phi$ be a completion of $\Delta$; we will use the same symbol $\Phi$ to denote its restriction to $\Sigma$. In this section, we define functors

$$
\mathbf{D}(\Sigma) \underset{i^{*}, i^{!}}{\stackrel{i_{*}, i_{!}}{\rightleftarrows}} \mathbf{D}(\Delta), \quad \mathbf{D}_{\Phi}(\Sigma) \underset{i^{*}, i^{!}}{\stackrel{i_{*}, i_{!}}{\rightleftarrows}} \mathbf{D}_{\Phi}(\Delta)
$$

satisfying familiar properties from sheaf theory. 
We begin by remarking that any locally free object $S \in \Lambda_{\Delta}$-mod has a bounded functorial resolution $S \rightarrow I^{\bullet}(S)$ by injective objects; just iterate the construction of (3.1.2).

We define the first four functors in (3.3.1) as follows:

- The extension functor $i_{*}$ sends injectives to injectives, so we define $i_{*}$ of a complex $J^{\bullet}$ to be the complex $i_{*} J^{\bullet}$.

- The restriction functor $\left.\right|_{\Sigma}: \Lambda_{\Delta}$-mod $\rightarrow \Lambda_{\Sigma}$-mod does not take injectives to injectives, so it must be derived: let $i^{*}$ of a complex $S^{\bullet}$ be the total complex of the double complex $I^{\bullet}\left(\left.J^{\bullet}\right|_{\Sigma}\right)$. (Note that if $\Sigma$ has a unique smallest cone, it is not necessary to derive this functor; this is the only case we will actually need.)

- Similarly, we define $i_{!}$by deriving the extension by zero functor $i_{! !}: \Lambda_{\Sigma}$-mod $\rightarrow \Lambda_{\Delta}$-mod. In other words, we set $i_{!} J^{\bullet}=$ total complex of $I \bullet\left(i_{! !} J^{\bullet}\right)$.

- To define $i^{!}$, let $\bar{\Sigma}$ be the closure of $\Sigma$ in $\Delta$. Given a sheaf $S \in \Lambda_{\Delta}$-mod and an open subset $U \subset \Delta$ define $\Gamma_{\bar{\Sigma}}(S ; U)$ to be the sections of $S$ on $U$ with support contained in $\bar{\Sigma}$. The map $U \mapsto \Gamma_{\bar{\Sigma}}(S ; U)$ defines a sheaf $S_{\bar{\Sigma}}$ on $\Delta$, and the functor $S \mapsto S_{\bar{\Sigma}}$ sends injectives to injectives. Then for a complex $J^{\bullet}$ in $\mathbf{D}(\Sigma)$, let $i^{!} J^{\bullet}=i^{*} J_{\bar{\Sigma}}^{\bullet}$

Suppose $\Delta$ has a completion $\Phi$ and $\Sigma$ has the induced completion. It is easy to check that the functors $i_{*}, i_{! !},\left.\right|_{\Sigma},(\cdot)_{\bar{\Sigma}}$, and $I^{\bullet}$ all extend in an obvious way to $\Phi$-stable sheaves and complexes, giving the other four functors in (3.3.1).

The functors $i^{*}, i^{!}, i_{!}, i_{*}$ and their stable versions satisfy the following standard properties for pushforward and pullback functors (see GM]); we leave their proof as an exercise for the reader:

(1) All four functors are exact functors of triangulated categories.

(2) If $\Pi \subset \Sigma$ with inclusion $j$, there are natural isomorphisms $j^{*} i^{*} \simeq(i \circ j)^{*}$, $i_{*} j_{*} \simeq(i \circ j)_{*}$, and similarly for $i^{!}, i_{!}$.

(3) $i^{*}$ is left adjoint to $i_{*}$, and $i^{!}$is right adjoint to $i_{!}$.

(4) There is a natural transformation $i^{!} \rightarrow i^{*}$. If $\Sigma \subset \Delta$ is open, it is an isomorphism.

(5) There is a natural transformation $i_{!} \rightarrow i_{*}$. If $\Sigma \subset \Delta$ is closed, it is an isomorphism.

For the remaining properties, assume $\Sigma$ is closed in $\Delta$, let $\Pi=\Delta \backslash \Sigma$, and let $j: \Pi \rightarrow \Delta$ be the inclusion.

(6) We have $j^{!} i_{*}=j^{*} i_{*}=0$.

(7) There are functorial distinguished triangles for $S^{\bullet}$ in $\mathbf{D}(\Delta)$ or $\mathbf{D}_{\Phi}(\Delta)$ :

$$
\begin{aligned}
& j ! j^{*} S^{\bullet} \rightarrow S^{\bullet} \rightarrow i_{*} i^{*} S^{\bullet} \rightarrow j_{!} j^{*} S^{\bullet}[1], \\
& i_{*} i^{!} S^{\bullet} \rightarrow S^{\bullet} \rightarrow j_{*} j^{*} S^{\bullet} \rightarrow i_{*} i^{!} S^{\bullet}[1] .
\end{aligned}
$$

(8) The adjunction morphisms

$$
\begin{aligned}
& i^{*} i_{*} S^{\bullet} \rightarrow S^{\bullet} \rightarrow i^{!} i_{*} S^{\bullet}, \\
& j^{*} j_{*} \widetilde{S}^{\bullet} \rightarrow \widetilde{S}^{\bullet} \rightarrow j^{*} j_{!} \widetilde{S}^{\bullet}
\end{aligned}
$$

are isomorphisms if $S^{\bullet} \in \mathbf{D}(\Sigma)$ or $\mathbf{D}_{\Phi}(\Sigma), \widetilde{S}^{\bullet} \in \mathbf{D}(\Pi)$ or $\mathbf{D}_{\Phi}(\Pi)$. 
3.4. The local $t$-structure. To simplify notation, we put $\mathbf{D}(\sigma)=\mathbf{D}(\{\sigma\})$ and $\mathbf{D}_{\Phi}(\sigma)=\mathbf{D}_{\Phi}(\{\sigma\})$ for any cone $\sigma$.

The properties (1)-(8) of the previous section are exactly what is needed to construct a $t$-structure on $\mathbf{D}(\Delta)$ by gluing "local" $t$-structures on the categories $\mathbf{D}(\sigma)$ for all $\sigma \in \Delta$. For more details on $t$-structures and gluing, see [BBD, GM], KS.

To define these local $t$-structures, take $\sigma \in \Delta$, and let $\mathbf{D}^{\geq 0}(\sigma)$ (respectively $\left.\mathbf{D}^{\leq 0}(\sigma)\right)$ be the full subcategory of objects $S^{\bullet}$ in $\mathbf{D}(\sigma)$ for which $\Gamma_{\sigma}\left(S^{\bullet}\right)_{q}^{p}=0$ if $p+q<-\operatorname{codim}(\sigma)($ resp. if $p+q>-\operatorname{codim}(\sigma))$.

3.4.1. Proposition. This defines a t-structure on $\mathbf{D}(\sigma)$.

Proof. Checking most of the axioms for a $t$-structure is fairly routine. We describe the hardest part, which is to show that for any $S^{\bullet} \in \mathbf{D}(\sigma)$ there is a distinguished triangle

$$
S_{\leq 0}^{\bullet} \rightarrow S^{\bullet} \rightarrow S_{\geq 1}^{\bullet} \rightarrow S_{\leq 0}^{\bullet}[1]
$$

with $S_{\leq 0}^{\bullet} \in \mathbf{D}^{\leq 0}(\sigma)$ and $S_{\geq 1}^{\bullet} \in \mathbf{D}^{\geq 1}(\sigma)=\mathbf{D}^{\geq 0}(\sigma)[-1]$. To show this, suppose that $S^{\bullet}=\left(\bar{S}_{q}^{p}, d\right)$, and let $S_{\leq 0}^{\bullet}$ be the subcomplex generated under the action of $\Lambda_{\sigma}$ by

(1) all elements of $S_{q}^{p}$, if $p+q \leq-\operatorname{codim}(\sigma)$, and

(2) elements of $S_{q}^{p}$ which are in the image of $d$, if $p+q=-\operatorname{codim}(\sigma)+1$.

We then let $S_{\geq 1}^{\bullet}$ be the quotient complex $S^{\bullet} / S_{\leq 0}^{\bullet}$. It is easy to see that $S_{\leq 0}^{\bullet}$ is a complex of free $\Lambda_{\sigma}$-modules, so $S_{\geq 1}^{\bullet}$ is also. The exact sequence

$$
0 \rightarrow S_{\leq 0}^{\bullet} \rightarrow S^{\bullet} \rightarrow S_{\geq 1}^{\bullet} \rightarrow 0
$$

induces the required distinguished triangle.

The core $\mathbf{P}(\sigma)=\mathbf{D}^{\geq 0}(\sigma) \cap \mathbf{D}^{\leq 0}(\sigma)$ of this $t$-structure is an artinian abelian category. If $k \in \mathbb{Z}$, the functor $\langle k\rangle=[k / 2]\{-k / 2\}$ is a $t$-exact automorphism of $\mathbf{D}(\sigma)$, and hence induces an automorphism of $\mathbf{P}(\sigma)$. All simple objects of $\mathbf{P}(\sigma)$ are of the form $\Lambda_{\sigma}[\operatorname{codim} \sigma]\langle k\rangle, k \in \mathbb{Z}$.

Given a completion $\Phi$, the $t$-structure on $\mathbf{D}_{\Phi}(\sigma)$ is defined by using the same vanishing restrictions for $\Gamma_{\sigma} F_{\sigma}$. The fact that this gives a $t$-structure is trivial, using Lemma 3.2.4.

3.5. The global $t$-structure. Given a quasifan $\Delta$, define a $t$-structure on $\mathbf{D}(\Delta)$ by

$$
\begin{gathered}
S^{\bullet} \in \mathbf{D}^{\geq 0}(\Delta) \Longleftrightarrow i_{\sigma}^{!}\left(S^{\bullet}\right) \in \mathbf{D}^{\geq 0}(\sigma) \text { for all } \sigma \in \Delta, \text { and } \\
S^{\bullet} \in \mathbf{D}^{\leq 0}(\Delta) \Longleftrightarrow i_{\sigma}^{*}\left(S^{\bullet}\right) \in \mathbf{D}^{\leq 0}(\sigma) \text { for all } \sigma \in \Delta .
\end{gathered}
$$

The $t$-structure on $\mathbf{D}_{\Phi}(\Delta)$ is defined similarly. The fact that these are $t$-structures follows from the standard arguments in $[\mathrm{BBD}$ ] and properties (1)-(8) from 93.3 ,

The cores of these $t$-structures are abelian categories, which we denote $\mathbf{P}(\Delta)$, $\mathbf{P}_{\Phi}(\Delta)$. Note that an object $S^{\bullet}$ is in $\mathbf{P}_{\Phi}(\Delta)$ if and only if its image under $F_{\Delta}$ is in $\mathbf{P}(\Delta)$. For any $k \in \mathbb{Z},\langle k\rangle=[k / 2]\{-k / 2\}$ is a $t$-exact automorphism of both $\mathbf{D}(\Delta)$ and $\mathbf{D}_{\Phi}(\Delta)$, and thus restricts to automorphisms of $\mathbf{P}(\Delta)$ and $\mathbf{P}_{\Phi}(\Delta)$.

3.5.1. Proposition. The forgetful functor $F_{\Delta}$ makes $\mathbf{P}_{\Phi}(\Delta)$ into a full subcategory of $\mathbf{P}(\Delta)$. 
Before proving this, we introduce two useful spectral sequences. Given objects $A_{0}^{\bullet}, B_{0}^{\bullet} \in \mathbf{D}(\Delta)$, the Hom-spaces $H^{k}=\operatorname{Hom}_{\mathbf{D}(\Delta)}^{k}\left(A_{0}^{\bullet}, B_{0}^{\bullet}\right)$ are the cohomology groups of a complex $C^{\bullet}$, with $C^{k}=\bigoplus_{l \in \mathbb{Z}} \operatorname{Hom}_{I n j_{\Delta}}\left(A_{0}^{l}, B_{0}^{k+l}\right)$. Filtering this complex by the support of the maps gives a spectral sequence with $E_{1}$ term

$$
E_{1}^{p, q}=\bigoplus_{\operatorname{dim} \sigma=p} \operatorname{Hom}_{\mathbf{D}(\sigma)}^{p+q}\left(i_{\sigma}^{*} A_{0}^{\bullet}, i_{\sigma}^{!} B_{0}^{*}\right)
$$

which abuts to $H^{p+q}$.

In a similar way, if $A^{\bullet}, B^{\bullet} \in \mathbf{D}_{\Phi}(\Delta)$, we have a spectral sequence with $E_{1}$ term

$$
{ }_{\Phi} E_{1}^{p, q}=\bigoplus_{\operatorname{dim} \sigma=p} \operatorname{Hom}_{\mathbf{D}_{\Phi}(\sigma)}^{p+q}\left(i_{\sigma}^{*} A^{\bullet}, i_{\sigma}^{!} B^{\bullet}\right)
$$

which abuts to $H_{\Phi}^{p+q}=\operatorname{Hom}_{\mathbf{D}(\Delta)}^{p+q}\left(A^{\bullet}, B^{\bullet}\right)$.

If $A_{0}^{\bullet}=F_{\Delta} A^{\bullet}, B_{0}^{\bullet}=F_{\Delta} B^{\bullet}$, then there is a natural map ${ }_{\Phi} E_{\bullet}^{p, q} \rightarrow E_{\bullet}^{p, q}$ of spectral sequences, whose $E_{1}$ component is induced by the forgetful functors $F_{\sigma}$, and which abuts to the map $F_{\Delta}: H_{\Phi}^{p+q} \rightarrow H^{p+q}$.

Proof of Proposition 3.5.1. Suppose $A^{\bullet}, B^{\bullet} \in \mathbf{P}_{\Phi}(\Delta)$, and take $A_{0}^{\bullet}, B_{0}^{\bullet}$ as above. Since $i_{\sigma}^{*} A^{\bullet} \in \mathbf{D}_{\Phi}^{\leq 0}(\sigma)$ and $i_{\sigma}^{!} B^{\bullet} \in \mathbf{D}_{\Phi}^{\geq 0}(\sigma)$, we have $E_{1}^{p, q}=0$ and ${ }_{\Phi} E_{1}^{p, q}=0$ if $p+q<0$. As a result, it is enough to prove that if $\sigma \in \Delta$ and $A^{\bullet} \in \mathbf{D}_{\Phi}^{\leq 0}(\sigma), B^{\bullet} \in$ $\mathbf{D}_{\Phi}^{\geq 0}(\sigma)$, then the natural map $F_{\sigma}: \operatorname{Hom}_{\mathbf{D}_{\Phi}(\sigma)}^{k}\left(A^{\bullet}, B^{\bullet}\right) \rightarrow \operatorname{Hom}_{\mathbf{D}(\sigma)}^{k}\left(F_{\sigma} A^{\bullet}, F_{\sigma} B^{\bullet}\right)$ is an injection for all $k$ and an isomorphism if $k=0$.

The injectivity follows immediately from Lemma 3.2.4. For surjectivity when $k=0$, note that by replacing $A^{\bullet}, B^{\bullet}$ by quasi-isomorphic complexes, we can assume that $A^{d}$ is generated in degrees $\leq-\operatorname{dim}(\sigma)-d$, while $B^{d}$ is generated in degrees $\geq-\operatorname{dim}(\sigma)-d$.

3.6. The middle extension. By standard arguments of [BBD, we have perverse extension functors

$$
j_{!_{*}}: \mathbf{P}(\Sigma) \rightarrow \mathbf{P}(\Delta), j_{!_{*}}: \mathbf{P}_{\Phi}(\Sigma) \rightarrow \mathbf{P}_{\Phi}(\Delta)
$$

defined for any inclusion $j: \Sigma \rightarrow \Delta$ of quasifans. They satisfy $(i j)_{!_{*}}=i_{!_{*}} j_{!_{*}}$ for any inclusion $i: \Delta \rightarrow \Pi$ of quasifans.

Perverse extension takes simple objects to simple objects. For $\sigma \in \Delta$, define $L_{\sigma}^{\bullet}=i_{\sigma ! *} \Lambda_{\sigma}[c / 2]\{c / 2\}$, where $c=\operatorname{codim}(\sigma)$; since $\mathbf{P}_{\Phi}(\Delta)$ is a full subcategory of $\mathbf{P}(\Delta)$, we use the same notation in both categories. All simple objects are isomorphic to $L_{\sigma}^{\bullet}\langle k\rangle$, for some $\sigma \in \Delta$ and $k \in \mathbb{Z}$.

There are two descriptions of $j_{1 *}$ which will be useful. If $\Sigma$ is closed in $\Delta$, then $j_{! *}=j_{!}=j_{*}$, so it is enough to let $\Sigma$ be an open set. Let $\Pi$ be the complementary closed set with inclusion $i: \Pi \rightarrow \Delta$.

3.6.1. Proposition. $j_{! *}$ is uniquely characterized by the following properties:

(1) $j^{*} j_{!_{*}}$ is the identity functor.

(2) For any $S^{\bullet} \in \mathbf{P}(\Sigma), i^{!} j_{! *} S^{\bullet} \in \mathbf{D}^{\leq-1}(\Pi)$ and $i^{*} j_{! *} S^{\bullet} \in \mathbf{D}^{\geq 1}(\Pi)$.

To give a more constructive description of $j_{! *}$, we restrict further to the case where $\Pi=\{\sigma\}$ is a single cone. Then $j_{!_{*}} S^{\bullet}[1]$ is the cone of the composed morphism

$$
j_{*} S^{\bullet} \rightarrow i_{\sigma *} i_{\sigma}^{*} j_{*} S^{\bullet} \rightarrow i_{\sigma *} \tau_{\geq 0} i_{\sigma}^{*} j_{*} S^{\bullet} .
$$


Remark. The object $L_{o}^{\bullet}$ constructed in this way is essentially a graded version of the combinatorial intersection cohomology complex constructed by McConnell in [M], although he expresses it as a sheaf of complexes rather than a complex of sheaves. His construction also works for more general perversities.

3.7. Costandard and injective perverse objects. Next we discuss costandard and injective objects in $\mathbf{P}_{\Phi}(\Delta)$. The costandard objects are particularly interesting, since although they contain many simple perverse objects in their composition series, when considered as complexes of injectives, they are as simple as possible.

Take $\sigma \in \Delta$, and let $c=\operatorname{codim} \sigma$. We define $N_{\sigma}^{\bullet}=j_{\sigma *} \Lambda_{\sigma}[c / 2]\{c / 2\}$. It is simply the injective object $J_{\sigma} \in I n j_{\Delta, \Phi}$, placed in degree $-c / 2$ and grading $-c / 2$. It is easy to see that it is perverse, since $j_{\tau}^{!} N_{\sigma}^{\bullet}=0$ if $\sigma \neq \tau$, while if $\sigma \prec \tau$, then $j_{\tau}^{*} N^{\bullet}=\Lambda_{\sigma}[c / 2]\{c / 2\}$, and $\Lambda_{\sigma}$, considered as a $\Lambda_{\tau}$-module, is generated in degrees $0 \leq d \leq \operatorname{dim} \tau-\operatorname{dim} \sigma$. We call the twists $N_{\sigma}^{\bullet}\langle k\rangle, k \in \mathbb{Z}$, costandard objects.

Dually, we also have standard objects $M_{\sigma}^{\bullet}\langle k\rangle$, where $M_{\sigma}^{\bullet}=j_{\sigma !} \Lambda_{\sigma}[c]$. They are also perverse; one way to see this would be to define a Verdier duality functor on $\mathbf{D}_{\Phi}(\Delta)$ and show that it preserves perversity and interchanges $j_{\text {! }}$ and $j_{*}$. It is easy to check perversity directly, however, since a minimal complex representing $M_{\sigma}^{\bullet}$ has

$$
M_{\sigma}^{i} \cong \bigoplus_{\substack{\sigma \prec \tau \\ \operatorname{codim} \tau=-i}} j_{\tau_{*}} \Lambda_{\sigma}
$$

which implies the required vanishing condition on $j_{\tau}^{!} M_{\sigma}^{\bullet}$, while $j_{\tau}^{*} M^{\bullet}=0$ if $\tau \neq \sigma$.

3.7.1. Theorem. The category $\mathbf{P}_{\Phi}(\Delta)$ has enough injectives; each injective object has a filtration by costandard objects.

This result is essentially the same as [BGS, Theorem 3.2.1]. In $\$ 4.4$ we will give an algorithm based on [BGS] which constructs the indecomposable injective objects as successive extensions of costandards. We do not give the proof that this algorithm works, as it is identical to the proof from [BGS, with two minor modifications. First, their proof is stated for projective objects and standard objects rather than injective objects and costandards, so one must dualize everywhere. Second, their proof works in an ungraded setting in which there is only one standard and one costandard object for each element in a finite poset, whereas our standards and costandards can each be twisted by $\langle k\rangle$ for any $k \in \mathbb{Z}$; instead of extending by a single costandard, therefore, one must extend by all possible twists.

All but one of the hypotheses of [BGS, Theorem 3.2.1] hold for formal reasons in any category of perverse sheaves in which lower star and lower shriek extensions from single strata are $t$-exact. The only one which requires explanation is the following.

3.7.2. Lemma. For any $\tau, \rho \in \Delta$ and $k \in \mathbb{Z}$, we have

$$
\operatorname{Ext}_{\mathbf{P}_{\Phi}(\Delta)}^{2}\left(M_{\tau}^{\bullet}, N_{\rho}^{\bullet}\langle k\rangle\right)=0 .
$$

Proof. First note that there is an injection (see [BGS, Lemma 3.2.4])

$$
\operatorname{Ext}_{\mathbf{P}_{\Phi}(\Delta)}^{2}\left(M_{\tau}^{\bullet}, N_{\rho}^{\bullet}\langle k\rangle\right) \rightarrow \operatorname{Hom}_{\mathbf{D}_{\Phi}(\Delta)}^{2}\left(M_{\tau}^{\bullet}, N_{\rho}^{\bullet}\langle k\rangle\right),
$$

so it will be enough to show that this second group is zero. This is equivalent to showing $\operatorname{Hom}^{2}\left(i_{\rho}^{*} M_{\tau}^{\bullet}, i_{\rho}^{*} N_{\rho}^{\bullet}\langle j\rangle\right)=0$. If $\tau \neq \rho$, we have $i_{\rho}^{*} M_{\tau}^{\bullet}=0$, so we are done. If $\tau=\rho$, use Lemma 3.2.4 
3.7.3. Theorem. There is an equivalence of categories

$$
\mathbf{D}_{\Phi}(\Delta) \cong D^{b}\left(\mathbf{P}_{\Phi}(\Delta)\right)
$$

in particular, $\operatorname{Hom}_{\mathbf{D}_{\Phi}(\Delta)}^{j}(A, B)=\operatorname{Ext}_{\mathbf{P}_{\Phi}(\Delta)}^{j}(A, B)$ for any $A, B \in \mathbf{P}_{\Phi}(\Delta)$.

Proof. See [BGS, Corollary 3.3.2].

It is also true that $\mathbf{D}(\Delta) \cong D^{b}(\mathbf{P}(\Delta))$, but the proof is more involved, and we do not need it here.

3.8. Example. We will illustrate the perverse $t$-structure on $\mathbf{D}(\Delta)$ and $\mathbf{D}_{\Phi}(\Delta)$ in the simplest nontrivial case. Let $V=\mathbb{R}$, and let $\sigma=\mathbb{R}_{\geq 0}, \Delta=[\sigma]=\{o, \sigma\}$. This is the fan of the toric variety $X_{\Delta}=\mathbb{C}$, with the orbit stratification $O_{\sigma}=\{0\}$, $O_{o}=\mathbb{C}^{*}$.

The reader may want to compare the statements that follow with the note BGSch by Beilinson, Ginzburg, and Schechtman, which explains the Koszul duality of $\overline{B G S}$ in the special case $\mathfrak{g}=\mathfrak{s l}_{2}$, where the flag variety is the projective line $\mathbb{P}^{1}$, with stratification $\left\{\{0\}, \mathbb{P}^{1} \backslash\{0\}\right\}$.

There are two indecomposable injectives in $\Lambda_{\Delta}$-mod, up to shifts of grading: $J_{o}=i_{o *} \Lambda_{o}$ and $J_{\sigma}=i_{\sigma *} \Lambda_{\sigma}$. The automorphism groups of both these objects are the scalars $\mathbb{R}$, and we have

$$
\operatorname{Hom}\left(J_{o}, J_{o}\{1\}\right) \cong \operatorname{Hom}\left(J_{o}, J_{\sigma}\right) \cong \operatorname{Hom}\left(J_{o}, J_{\sigma}\{-1\}\right) \cong \mathbb{R} .
$$

For any other $\rho, \tau \in \Delta$ and $k \in \mathbb{Z}$, we have $\operatorname{Hom}\left(J_{\rho}, J_{\tau}\{k\}\right)=0$.

\begin{tabular}{|c|c|c|c|}
\hline$S^{\bullet}$ & $S^{-1} \rightarrow S^{0}$ & $\operatorname{real}_{\Delta} S^{\bullet}$ & $A_{o} \underset{q}{\stackrel{p}{\rightleftarrows}} A_{\sigma}$ \\
\hline$L_{\sigma}^{\bullet}=N_{\sigma}^{\bullet}$ & $0 \rightarrow J_{\sigma}$ & $i_{\sigma ! *} \mathbb{R}_{\sigma}$ & $0 \underset{0}{\stackrel{0}{\rightleftarrows}} \mathbb{R}$ \\
\hline$N_{o}^{\bullet}\langle 1\rangle=I_{o}^{\bullet}\langle 1\rangle$ & $J_{o} \rightarrow 0$ & $i_{o *} \mathbb{R}_{O}[1]$ & $\mathbb{R} \underset{1}{\stackrel{0}{\rightleftarrows}} \mathbb{R}$ \\
\hline$L_{o}^{\bullet}\langle 1\rangle$ & $J_{o} \rightarrow J_{\sigma}\{-1\}$ & $i_{o ! *} \mathbb{R}_{o}[1]$ & $\mathbb{R} \underset{0}{\stackrel{0}{\rightleftarrows} 0} 0$ \\
\hline$M_{o}^{\bullet}\langle 1\rangle$ & $J_{o} \rightarrow J_{\sigma} \oplus J_{\sigma}\{-1\}$ & $i_{o !} \mathbb{R}_{o}[1]$ & $\mathbb{R} \underset{0}{\stackrel{1}{\rightleftarrows}} \mathbb{R}$ \\
\hline$I_{\sigma}^{\bullet}$ & $J_{o} \rightarrow J_{\sigma}$ & $\mathcal{I}$ & $\mathbb{R} \underset{(1,0)}{\stackrel{\left(\begin{array}{l}0 \\
1\end{array}\right)}{\rightleftarrows}} \mathbb{R}^{2}$ \\
\hline
\end{tabular}

TABLE 1. Some perverse objects

In the first two columns of Table 1 we list five objects in $\mathbf{P}(\Delta)$, along with the names we have been using for them (the use of $I_{\sigma}^{\bullet}$ and $I_{o}^{\bullet}$ is potentially misleading, as these objects are not injective in $\mathbf{P}(\Delta)$; they are the images of the corresponding injectives in $\mathbf{P}_{\Phi}(\Delta)$ under $F_{\Delta}$ ). In the second column, the left element in each complex is placed in degree -1 , and all maps have maximal possible rank.

In the third column we list the corresponding perverse sheaves on $X_{\Delta}$ obtained by applying the functor real ${ }_{\Delta}$ of Theorem 1.4.2, Here $\mathbb{R}_{\tau}=\mathbb{R}_{O_{\tau}}$, the constant rank one local system on $O_{\tau}$, for $\tau=o, \sigma$. We abuse notation and write $i_{\tau}$ for the inclusion of $O_{\tau}$ into $X_{\Delta}$. 
The perverse sheaf $\mathcal{I}$ is the largest indecomposable extension of $\mathbb{R}_{o}[1]$; it fits in an exact sequence

$$
0 \rightarrow i_{\sigma ! *} \mathbb{R}_{\sigma} \rightarrow \mathcal{I} \rightarrow i_{o *} \mathbb{R}_{o}[1] \rightarrow 0 .
$$

This and other standard exact sequences involving these perverse sheaves can be easily constructed in $\mathbf{D}(\Delta)$, as the reader is invited to check.

In the fourth column we describe these perverse sheaves in terms of the following well-known result.

3.8.1. Theorem $([\mathrm{MV}, \overline{\mathrm{V}}])$. The category of perverse sheaves on $\mathbb{C}$ constructible with respect to the stratification $\left\{\{0\}, \mathbb{C}^{*}\right\}$ is equivalent to the category of finitedimensional vector spaces and maps

$$
M_{o} \stackrel{p}{\stackrel{p}{\rightleftarrows}} M_{\sigma}
$$

for which $1+q p$ is invertible. Under this equivalence, the vector space $M_{o}$ is the stalk cohomology at a point of $O_{o}=\mathbb{C}^{*}$, and the operator $1+q p$ gives the monodromy action of a loop around 0.

There is a nonzero nilpotent map $\mathcal{I} \rightarrow \mathcal{I}$. It is the image under real $\Delta$ of the following map of complexes (all maps have maximal possible rank):

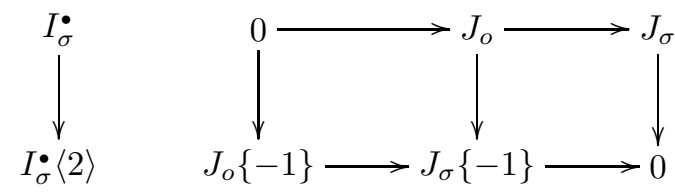

It is easy to see that this is not chain-homotopic to 0 .

3.9. Example of stable sheaves. The fan $\Delta$ from the previous section has a unique completion given by $\Phi_{o}=\{0\}, \Phi_{\sigma}=V^{*}$. Passing from $\mathbf{D}(\Delta)$ to $\mathbf{D}_{\Phi}(\Delta)$ corresponds intuitively to embedding $X_{\Delta}=\mathbb{C}$ into $\mathbb{P}^{1}$ with strata $O_{\tau}^{\prime}=\overline{O_{\tau}}, \tau=$ $o, \sigma$. These new strata have the same local properties as before, but now the open orbit is contractible.

An orbit-constructible perverse sheaf $P$ on $\mathbb{C}$ extends to a perverse sheaf on $\mathbb{C P}^{1}$ constructible with respect to this stratification if and only if $H^{-1}\left(\left.P\right|_{O_{o}}\right)$ is a trivial local system. There are five isomorphism classes of indecomposable perverse sheaves on $X_{\Delta}$ with this property, namely the five in Table 1 . On the other hand, the objects in $\mathbf{P}(\Delta)$ from the left column of the table all have a unique lift to $\mathbf{P}_{\Phi}(\Delta)$, and they are the only indecomposable objects which lift.

The category $\mathbf{P}_{\Phi}(\Delta)$ is a mixed version of the category $\mathcal{O}$ for $\mathfrak{g}=\mathfrak{s l}_{2}$. The statement of Koszul duality in this case is identical with [BGS].

Note that the nonzero map $I_{\sigma}^{\bullet} \rightarrow I_{\sigma}^{\bullet}\langle 2\rangle$ described above lifts uniquely to a nonzero map in $\mathbf{D}_{\Phi}(\Delta)$. Since $\sigma$ is isomorphic to its own dual cone $\sigma^{\vee}$, the properties of the Koszul duality functor asserted by Theorem 1.2.1 imply that there should be a nonzero map

$$
L_{o}^{\bullet} \rightarrow L_{o}^{\bullet}[2]\langle-2\rangle=L_{o}^{\bullet}[1]\{1\}
$$


in $\mathbf{D}_{\Phi}(\Delta)$. Indeed, we have the following map of complexes:

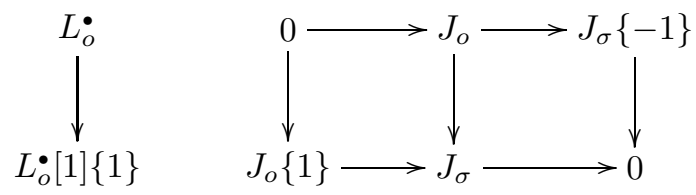

Note that this map becomes zero in $\mathbf{D}(\Delta)$, so $F_{\Delta}: \mathbf{D}_{\Phi}(\Delta) \rightarrow \mathbf{D}(\Delta)$ is not a full embedding, although its restriction to $\mathbf{P}_{\Phi}(\Delta)$ is.

\section{Koszul duality}

4.1. The dual cone. Fix a cone $\sigma \subset V$. The dual cone $\sigma^{\vee} \subset V^{*}$ is given by

$$
\left\{\xi \in V^{*} \mid \xi(v) \geq 0 \text { for all } v \in \sigma\right\} .
$$

There is an order-reversing bijection $\tau \mapsto \tau^{\perp}$ between faces of $\sigma$ and faces of $\sigma^{\vee}$, defined by

$$
\tau^{\perp}=\sigma^{\vee} \cap\left(V_{\tau}\right)^{\perp} .
$$

Fix a completion $\Phi$ on the fan $[\sigma]$, as described in 3.2 It induces a dual completion $\Phi^{\vee}$ on $\left[\sigma^{\vee}\right]$, by $\Phi_{\tau^{\perp}}^{\vee}=\left(\Phi_{\tau}\right)^{\perp}$. If $\sigma$ is a rational cone, then $\Phi$ is rational if and only if $\Phi^{\vee}$ is rational.

4.2. The Koszul functor. Stated in terms of the combinatorial categories of $\S 3$, our main theorem is the following.

4.2.1. Theorem. Given an n-dimensional pointed cone $\sigma \subset V \cong \mathbb{R}^{n}$ and a combinatorial completion $\Phi$ of $[\sigma]$, there is an equivalence of triangulated categories

$$
\kappa: \mathbf{D}_{\Phi}([\sigma])^{o p p} \rightarrow \mathbf{D}_{\Phi \vee}\left(\left[\sigma^{\vee}\right]\right)
$$

for which

(a) $\kappa(M \bullet[k])=\left(\kappa M^{\bullet}\right)[-k]$ and $\kappa(M \bullet\langle k\rangle)=\left(\kappa M^{\bullet}\right)[-k]\langle k\rangle$ for any $M^{\bullet} \in$ $\mathbf{D}_{\Phi}([\sigma])$, and

(b) $\kappa\left(L_{\dot{\tau}}^{\bullet} \cong I_{\tau^{\perp}}\right.$ and $\kappa\left(I_{\tau}^{\bullet}\right) \cong L_{\tau^{\perp}}$ for any $\tau \prec \sigma$.

Here $I_{\tau}^{\bullet}, I_{\tau^{\perp}}$ are injective hulls of the simple objects $L_{\tau}^{\bullet}$ and $L_{\tau^{\perp}}$ in $\mathbf{P}_{\Phi}([\sigma])$ and $\mathbf{P}_{\Phi \vee}\left(\left[\sigma^{\vee}\right]\right)$, respectively.

We will define this functor as the derived functor of a contravariant functor

$$
K=K_{\sigma}: \Lambda_{[\sigma]}-\bmod _{\Phi} \rightarrow \Lambda_{\left[\sigma^{\vee}\right]}-\bmod _{\Phi^{\vee}} .
$$

Let $k$ denote the functor $\mathbb{R}$-mod $\rightarrow \mathbb{R}$-mod which reverses the grading, so $k(M)_{j}=M_{-j}$. Note that for any vector space $V$, interior multiplication makes $k\left(\Lambda\left(V^{*}\right)\right)$ into a graded $\Lambda(V)$-module.

Define the functor $K$ as follows. Take $S \in \Lambda_{[\sigma]}-\bmod _{\Phi}$. The stalk of $K S$ at a cone $\tau^{\perp} \in\left[\sigma^{\vee}\right]$ is given by

$$
(K S)_{\tau^{\perp}}=k\left(\Lambda\left(\Phi_{\tau}\right) \otimes \operatorname{hom}\left(J_{\tau}, S\right)^{*}\right)
$$

where hom $=\operatorname{hom}_{\Lambda_{[\sigma]}-\bmod _{\Phi}}$ is the graded hom of $\$ 2.1$ and $\otimes$ denotes the tensor product of graded $\mathbb{R}$-modules. It has an action of $\Lambda_{\tau^{\perp}}=\Lambda\left(V_{\tau}\right)$ by interior multiplication on the first factor and the trivial action on the second. It acquires a 
stabilization by setting

$$
(K S)_{\tau^{\perp}}^{\natural}=k\left(\omega_{\tau} \otimes \operatorname{hom}\left(J_{\tau}, S\right)^{*}\right),
$$

where $\omega_{\tau}=\Lambda^{\operatorname{dim} \tau}\left(\Phi_{\tau}\right) \cong \mathbb{R}\{-\operatorname{dim} \tau\}$.

To make these stalks into a sheaf, we must define restriction maps

$$
r_{\rho^{\perp}, \tau^{\perp}}:(K S)_{\tau^{\perp}} \rightarrow(K S)_{\rho^{\perp}}
$$

for $\tau \prec \rho$. Note that Proposition 3.2.5 says that hom $\left(J_{\tau}, J_{\rho}\right)=\Lambda\left(\Phi_{\rho}^{\tau}\right)^{*}$ canonically, so we can dualize the composition homomorphism to get a map

$$
\operatorname{hom}\left(J_{\tau}, S\right)^{*} \rightarrow \Lambda\left(\Phi_{\rho}^{\tau}\right) \otimes \operatorname{hom}\left(J_{\rho}, S\right)^{*} .
$$

Exterior multiplication gives a map (in fact an isomorphism)

$$
\Lambda\left(\Phi_{\tau}\right) \otimes \Lambda\left(\Phi_{\rho}^{\tau}\right) \rightarrow \Lambda\left(\Phi_{\rho}\right) .
$$

The required map $r_{\rho^{\perp}, \tau^{\perp}}$ is obtained by combining (4.2.2) and (4.2.3). It is a homomorphism of $\Lambda_{\tau^{\perp-}}$-modules, since $\Lambda_{\tau^{\perp}}$ acts trivially on $\Lambda\left(\Phi_{\rho}^{\tau}\right)$.

Remark. One can also construct $K$ directly using the description of morphisms in $\operatorname{Inj}_{[\sigma], \Phi}$ given by Proposition 3.2.5.

4.2.4. Theorem. $K$ is a contravariant functor $\Lambda_{[\sigma]}-\bmod _{\Phi} \rightarrow \Lambda_{\left[\sigma^{\vee}\right]}-\bmod _{\Phi^{\vee}}$. It satisfies the following properties:

(1) $K$ is left exact; we have $K(S\{n\})=(K S)\{n\}$ for all sheaves $S$ and all $k \in \mathbb{Z}$.

(2) For any $\tau \in[\sigma]$, there is an isomorphism

$$
K J_{\tau} \cong J_{\tau^{\perp}}\{\operatorname{dim} \tau\} .
$$

(3) $K$ restricts to an isomorphism of categories

$$
\operatorname{Inj}_{[\sigma], \Phi} \rightarrow \operatorname{In} j_{\left[\sigma^{\vee}\right], \Phi \vee} .
$$

Proof. Using Proposition 3.2.5, it is easy to see that $r_{\rho^{\perp}, \tau^{\perp}} r_{\tau^{\perp}, \mu^{\perp}}=r_{\rho^{\perp}, \mu^{\perp}}$ whenever $\mu \prec \rho \prec \tau$, so $K S$ is a sheaf. It is stable, since

$$
r_{\rho^{\perp}, \tau^{\perp}}\left((K S)_{\tau^{\perp}}^{\natural}\right) \subset k\left(\left(\omega_{\tau} \cdot \Lambda\left(\Phi_{\rho}^{\tau}\right)\right) \otimes \operatorname{hom}\left(J_{\rho}, S\right)^{*}\right)=k\left(\Lambda\left(\left(\Phi^{\vee}\right)_{\tau^{\perp}}^{\rho^{\perp}}\right) \cdot(K S)_{\rho^{\perp}}^{\natural}\right),
$$

where the equality follows since $\left(\Phi^{\vee}\right)_{\tau^{\perp}}^{\rho^{\perp}}=\Phi_{\tau}^{\vee} \cap V_{\rho}$ is the annihilator of $\Phi_{\tau}$ in $V_{\rho}$.

Part (1) of the theorem is clear. To show (2), take $\rho^{\perp} \in\left[\sigma^{\vee}\right]$. If $\rho \nprec \tau$, then $\left(K J_{\tau}\right)_{\rho^{\perp}}=0$. If $\rho \prec \tau$, then Proposition 3.2.5 gives a canonical identification

$$
\begin{aligned}
\left(K J_{\tau}\right)_{\rho^{\perp}} & =k\left(\Lambda\left(\Phi_{\rho}\right) \otimes \operatorname{hom}\left(J_{\rho}, J_{\tau}\right)^{*}\right) \\
& \cong k\left(\Lambda\left(\Phi_{\rho}\right) \otimes \Lambda\left(\Phi_{\tau}^{\rho}\right)\right) \cong k\left(\Lambda\left(\Phi_{\tau}\right)\right) .
\end{aligned}
$$

The direct sum decomposition $V^{*}=\Phi_{\tau} \oplus V_{\tau}^{\perp}$ shows that $k\left(\Lambda\left(\Phi_{\tau}\right)\right)$ is isomorphic to $\Lambda_{\tau^{\perp}}$ as a $\Lambda_{\rho^{\perp}}$-module. It is easy to check that the restriction maps between these stalks are isomorphisms.

The essential surjectivity in (3) follows, so we only need to show full faithfulness. It will be enough to show that for $\tau \prec \rho$ the map

$$
\operatorname{Hom}_{\Lambda_{[\sigma]-\bmod }}\left(J_{\rho}, J_{\tau}\right) \rightarrow \operatorname{Hom}_{\Lambda_{[\sigma \vee}-\bmod }\left(K\left(J_{\tau}\right), K\left(J_{\rho}\right)\right)
$$

is injective, since both sides have the same dimension. But applying $\operatorname{hom}\left(J_{\rho},-\right)$ to a nonzero map $J_{\rho} \rightarrow J_{\tau}$ results in a nonzero map. 
Now, let $\tilde{\kappa}: \mathbf{D}_{\Phi}([\sigma]) \rightarrow \mathbf{D}_{\Phi^{\vee}}\left(\left[\sigma^{\vee}\right]\right)$ be the derived functor of $K:$ if $\left(I^{\bullet}, d\right)$ is a complex of injectives, then $\tilde{\kappa}\left(I^{\bullet}, d\right)=\left((K I)^{-\bullet}, K d\right)$. The functor $\kappa$ we want is then $\tilde{\kappa}$ followed by the twist $\langle\operatorname{dim} V\rangle$.

It follows from Theorem 4.2.4 that $\kappa$ is an equivalence of categories. It is also clear that part (a) of Theorem 4.2.1 holds. The proof of part (b) - that $\kappa^{\vee}$ interchanges simple and injective objects - will occupy the rest of $\S 4$. The key ingredient is the following result, which follows immediately from Theorem 4.2.4(2).

4.2.6. Lemma. For any $\tau \in[\sigma]$, there is an isomorphism $\kappa N_{\dot{\tau}}^{\bullet} \cong N_{\tau^{\perp}}$.

Remark. This isomorphism is not canonical, and the functor $\kappa_{\sigma^{\vee}}: \mathbf{D}_{\Phi^{\vee}}\left(\left[\sigma^{\vee}\right]\right) \rightarrow$ $\mathbf{D}_{\Phi}([\sigma])$ defined by switching the roles of $\sigma$ and $\sigma^{\vee}$ is not canonically the inverse of $\kappa$. The correct inverse functor is $\kappa^{\vee}=\omega_{\sigma} \otimes \kappa_{\sigma^{\vee}}$, where the one-dimensional vector space $\omega_{\sigma}$ is in bidegree $(0,0)$. With this definition, there is a canonical isomorphism $\kappa^{\vee} \kappa \simeq$ id.

Remark. It is possible to view $\kappa$ as a convolution functor, analogous to the geometric Fourier transform. There is a sheaf $\Xi$ on the poset $\left[\sigma \times \sigma^{\vee}\right]$ whose stalk on $\left(\tau, \rho^{\perp}\right)$ is $\Lambda_{\sigma}=\Lambda(V)$ if $\rho \prec \tau$ and is 0 otherwise. The support of $\Xi$ can be thought of as a "combinatorial conormal variety" to the fan $[\sigma]$.

Exterior and interior multiplication define commuting actions of $p_{1}^{*} \Lambda_{[\sigma]}$ and $p_{2}^{*} \Lambda_{\left[\sigma^{\vee}\right]}$ on $\Xi$, where $p_{1}$ and $p_{2}$ are the projections of $\left[\sigma \times \sigma^{\vee}\right]=[\sigma] \times\left[\sigma^{\vee}\right]$ onto the first and second factors. Then $\kappa$ can be written

$$
S^{\bullet} \mapsto p_{2 *} R \mathcal{H} \operatorname{om}_{p_{1}^{*} \Lambda_{[\sigma]}}\left(p_{1}^{*} S^{\bullet}, \Xi\right),
$$

where $p_{2 *}$ and $R \mathcal{H}$ om must be defined to take the completions $\Phi, \Phi^{\vee}$ into account.

It would be interesting to know if our Koszul duality functor can be described geometrically as a similar convolution on filtered $D$-modules.

4.3. Purity of the IC sheaves. We return for the moment to the case of a general quasifan $\Delta$ in $V$. We need the following result about the simple perverse sheaves $L_{\tau}^{\bullet}$.

4.3.1. Theorem. If $\tau \prec \sigma$ are cones in $\Delta$, and $j \in \frac{1}{2} \mathbb{Z}$, then $H^{j}\left(i_{\sigma}^{*} L_{\tau}^{\bullet}\right)$ and $H^{j}\left(i_{\sigma}^{!} L_{\boldsymbol{\tau}}\right)$ are free $\Lambda_{\sigma}$-modules generated in degree $j$.

To prove this, we import results from the theory of equivariant intersection cohomology of fans, developed independently in $[\mathrm{BBFK},[\mathrm{BrL}$. The key result is the Hard Lefschetz theorem proved by Karu [Ka]. We recall the basic facts of this theory.

Given a fan $\Delta$, let $\mathcal{A}=\mathcal{A}_{\Delta}$ denote the sheaf of $\Delta$-piecewise polynomial functions on $\bigcup_{\sigma \in \Delta} \sigma$, graded so that linear functions have degree two. In the language of 22.4 the stalk $\mathcal{A}_{\sigma}$ is the symmetric algebra $S\left(V_{\sigma}^{*}\right)$, with the the obvious restriction map $\mathcal{A}_{\sigma} \rightarrow \mathcal{A}_{\tau}$ if $\tau \prec \sigma$.

Since all of these rings are quotients of $A=S\left(V^{*}\right)$, any space of sections of an $\mathcal{A}$-module will be an $A$-module. For any $A$-module $M$, we define $\bar{M}=M \otimes_{A} \mathbb{R}$.

Let us fix a cone $\tau \in \Delta$. A minimal extension sheaf for $\tau$ on $\Delta$ is an $\mathcal{A}$-module $\mathcal{L}=\mathcal{L}^{\tau}$ satisfying:

(1) (normalization) $\mathcal{L}_{\tau} \cong \mathcal{A}_{\tau}\{\operatorname{codim} \tau\}$, and $\mathcal{L}_{\sigma}=0$ if $\tau \nprec \sigma$,

(2) (local freeness) $\mathcal{L}_{\sigma}$ is a free $\mathcal{A}_{\sigma}$-module for each $\sigma \in \Delta$, and 
(3) (minimal local extension) for each $\sigma \in \Delta, \sigma \neq \tau$, the homomorphism

$$
\overline{\mathcal{L}_{\sigma}} \rightarrow \overline{\mathcal{L}(\partial \sigma)}
$$

is an isomorphism of graded vector spaces.

Here we define $\partial \sigma=(\Delta \cap[\sigma]) \backslash\{\sigma\}$.

Such a sheaf always exists, and is unique up to a scalar isomorphism. The shift $\operatorname{codim} \tau$ in (1) does not appear in $[\mathrm{BBFK}]$ or [BrL; we have added it so the minimal extension sheaf is perverse.

Properties (1) and (3) imply that $\mathcal{L}_{\sigma} \rightarrow \mathcal{L}(\partial \sigma)$ is surjective for any $\sigma$, or in other words, that forgetting the $\mathcal{A}$-module structure makes $\mathcal{L}$ injective as a sheaf of vector spaces. Define $\mathcal{L}(\sigma, \partial \sigma)$ to be the kernel of $\mathcal{L}_{\sigma} \rightarrow \mathcal{L}(\partial \sigma)$.

4.3.2. Theorem (BBFK, $\mathrm{Ka})$. For every $\sigma \in \Delta, \sigma \neq \tau$, we have:

(a) $\mathcal{L}(\sigma, \partial \sigma)$ is a free $\mathcal{A}_{\sigma}$-module,

(b) $\overline{\mathcal{L}_{\sigma}}$ vanishes in degrees $\geq-\operatorname{codim} \sigma$, and

(c) $\overline{\mathcal{L}(\sigma, \partial \sigma)}$ vanishes in degrees $\leq-\operatorname{codim} \sigma$.

Proof. (a) is a special case of Corollary 4.12 of [BBFK. (b) is equivalent to (c) by Remark 1.8(ii) of [BBFK]. Finally, (b) is equivalent to the "Hard Lefschetz" theorem proved by Karu [Ka]: the implication HL $\Longrightarrow$ (b) is given in the last paragraph of BBFK, but their argument runs just as well backward. Note that most of these proofs are stated for the case of $\tau=o$, but they extend easily to the case of general $\tau$.

Let $\Lambda^{\vee}$ be the exterior algebra of $V$, graded so that $V$ has degree -1 . This ring is the Koszul dual to the polynomial ring $A$, in the sense of [BGG, BGS, GKM]. We will apply the BGG Koszul functor (specifically, the graded version of BGS]) to the stalks of $\mathcal{L}$ to obtain a complex of sheaves of $\Lambda^{\vee}$-modules.

For a graded $A$-module $M=\bigoplus M_{j}$, define a complex $h(M)=\left(N^{\bullet}, d\right)$, where $N^{\bullet}=M \otimes \Lambda^{\vee}$ with the grading given by placing $M_{j} \otimes \Lambda_{i}^{\vee}$ in complex degree $j / 2$ and grading degree $j / 2-i+\operatorname{dim} V$. The boundary map $d$ is given by choosing a basis $\left\{v_{i}\right\}$ for $V$ and the dual basis $\left\{v_{i}^{*}\right\}$ for $V^{*}$, and setting

$$
d(m \otimes \lambda)=\sum_{i} v_{i}^{*} m \otimes\left(\lambda \wedge v_{i}\right) .
$$

Given our minimal extension sheaf $\mathcal{L}=\mathcal{L}^{\tau}$, we define a sheaf of $\Lambda^{\vee}$-modules $S^{\bullet}=h(\mathcal{L})$ by $S_{\sigma}^{\bullet}=h\left(\mathcal{L}_{\sigma}\right)$. The following lemma implies that it has the structure of a complex of $\Lambda_{\Delta}$-modules.

4.3.3. Lemma. Suppose that $M$ is a free $\mathcal{A}_{\sigma}$-module, so that elements of $V_{\sigma}^{\perp}$ act trivially. Then we can give $h(M)$ the structure of a complex of $\Lambda_{\sigma}=\Lambda\left(V_{\sigma}^{\perp}\right)$ modules so that $\Gamma_{\sigma}(h(M))$ is isomorphic as a bigraded vector space to $\bar{M}$, with $\bar{M}_{j}$ appearing in bidegree $(j / 2, j / 2)$.

Proof. Let an element $a \in \Lambda_{\sigma}$ act via $a(m \otimes \lambda)=m \otimes i(a) \lambda$, where $i(a)$ denotes interior multiplication by $a$.

This gives a $\Lambda_{\sigma}$-module structure on the stalk $S_{\sigma}^{\bullet}$. It is clearly compatible with the maps between faces, so $S \bullet$ becomes a complex of $\Lambda_{\Delta}$-sheaves. Since $\mathcal{L}$ is injective as an $\mathbb{R}$-sheaf, so is $S^{\bullet}$. Using the second part of Lemma 4.3.3, we see that although $S^{\bullet}$ is only bounded below as a complex of injective $\Lambda_{\Delta}$-sheaves, its cohomology vanishes outside of a bounded set of degrees, so in fact $S^{\bullet} \in \mathbf{D}(\Delta)$. 
4.3.4. Lemma. We have $i_{\sigma}^{!} S^{\bullet}=h(\mathcal{L}(\sigma, \partial \sigma))$.

Proof. Split $\mathcal{L}$ into a direct sum of indecomposable injective $\mathbb{R}$-sheaves. The only ones which contribute to either side are the ones supported on the closure of $\sigma$.

Combining this with Lemma 4.3 .3 shows that $S^{\bullet}$ satisfies the same vanishing conditions of Proposition 3.6.1, and so $S^{\bullet} \cong L_{\tau}^{\bullet}$. Theorem 4.3 .1 now follows by using Theorem 4.3.2 and Lemma 4.3.3.

Remark. The construction actually gives a functor $\mathcal{A}_{\Delta}-\bmod _{\text {pure }} \rightarrow \mathbf{D}(\Delta)$, where $\mathcal{A}_{\Delta}$-mod $\operatorname{mure}_{\text {pur }}$ is the category of pure $\mathcal{A}$-modules, which are finite direct sums of shifts of minimal extension sheaves $\mathcal{L}^{\tau}$. In $[\mathrm{BL}$ complexes of pure sheaves are used to model $T$-equivariant mixed sheaves on toric varieties in the same way $\mathbf{D}(\Delta)$ models constructible mixed sheaves.

4.4. Injectives and simples. Now we prove part (b) of Theorem 4.2.1, which says that $\kappa$ interchanges simple and injective objects.

4.4.1. Constructing simples. We begin by restating the construction of simples from \$3.6 in a suitable form. Take a quasifan $\Delta$ and a face $\tau \in \Delta$ of codimension $c$; we want to construct the simple perverse object $L^{\bullet}=L_{\tau}^{\bullet}$. Refine the partial order on the cones in the closure $\bar{\tau}=\{\rho \in \Delta \mid \tau \prec \rho\}$ to a total order; this gives a sequence $\rho_{0}=\tau, \rho_{1}, \ldots, \rho_{N}$, so that the set $\Delta_{k}=(\Delta \backslash \bar{\tau}) \cup\left\{\rho_{0}, \ldots, \rho_{k}\right\}$ is open in $\Delta$ for $k=0, \ldots, N$. Let $j_{k}: \Delta_{k} \rightarrow \Delta$ be the inclusion.

The inductive construction of $L^{\bullet}$ constructs the objects $L_{k}=j_{k *} j_{k}^{*} L^{\bullet}$ for $k=$ $0, \ldots, N$, starting with $L_{0}^{\bullet}=N_{\tau}^{\bullet}$ and ending with $L_{N}^{\bullet}=L^{\bullet}$. Assuming $L_{\dot{k}-1}$ is already constructed, then $L_{\dot{k}}[1]$ is the cone of the map

$$
L_{k-1}^{\bullet} \rightarrow \tau\left(\operatorname{hom}^{\bullet}\left(L_{k-1}^{\bullet}, N_{\rho_{k}}^{\bullet}\right)^{*}\right) \otimes N_{\rho_{k}}^{\bullet},
$$

where $\tau$ is the truncation on bigraded vector spaces $M$ given by $\tau(M)_{q}^{p}=M_{q}^{p}$ for $p+q \geq 0$ and $\tau(M)_{q}^{p}=0$ for $p+q<0$, and the map is obtained from the natural maps $L_{k-1}^{\bullet} \rightarrow \operatorname{hom}^{\bullet}\left(L_{k-1}^{\bullet}, N_{k}^{\bullet}\right)^{*} \otimes N_{k}^{\bullet}$ and $M \rightarrow \tau(M)$.

So far this is just writing out the inductive computation of \$3.6. Using Theorem 4.3.1 we can alter the algorithm in the following way.

4.4.2. Theorem. The algorithm described above is unchanged if the truncation $\tau$ is replaced by $\tau^{\prime}$, which keeps only those $M_{q}^{p}$ for which $q=p+1$.

Proof. Let $\rho=\rho_{k}, r=\operatorname{codim} \rho / 2$. We have

$$
\begin{aligned}
\operatorname{hom}^{\bullet}\left(L_{k-1}^{\bullet}, N_{k}^{\bullet}\right)^{*} & =\operatorname{hom}^{\bullet}\left(j_{\rho}^{*} L_{k-1}^{\bullet}, \Lambda_{\rho}\{r\}[r]\right)^{*} \\
& =\Gamma_{\rho}\left(j_{\rho}^{*} L_{k-1}^{\bullet}\right)\{-r\}[-r] .
\end{aligned}
$$

The theorem thus follows from the following fact: $\Gamma_{\rho}\left(j_{\rho}^{*} L_{k-1}\right)_{q}^{p}=0$ unless either $q=p<r$ or $q=p+1>r$. To show this, let $i_{k}: \Delta \backslash \Delta_{k-1} \rightarrow \Delta$ be the inclusion. Applying $j_{\rho}^{*}$ to the triangle

$$
i_{k *} i_{k}^{!} L^{\bullet} \rightarrow L^{\bullet} \rightarrow L_{k-1}^{\bullet} \rightarrow i_{k *} i_{k}^{!} L^{\bullet}[1]
$$

gives a triangle

$$
j_{\rho}^{!} L^{\bullet} \rightarrow j_{\rho}^{*} L^{\bullet} \rightarrow j_{\rho}^{*} L_{k-1}^{\bullet} \rightarrow j_{\rho}^{!} L^{\bullet}[1] .
$$

The first map vanishes by the vanishing conditions for $L^{\bullet}$ and Lemma 3.2.4. Thus

$$
j_{\rho}^{*} L_{k-1} \cong j_{\rho}^{*} L^{\bullet} \oplus j_{\rho}^{!} L^{\bullet}[1] .
$$

The claim now follows from the vanishing conditions for $L^{\bullet}$ and Theorem 4.3.1. 
4.4.3. Constructing injectives. To complete the proof of Theorem 4.2.1(b), we give an algorithm for constructing injective objects in $\mathbf{D}_{\Phi}(\Delta)$ which is dual under the functor $\kappa$ to the construction of simples given above. For a proof that the following algorithm does indeed give an injective object, see [BGS, Theorem 3.2.1].

Take a cone $\sigma$ and a completion $\Phi$ of $[\sigma]$. Let $\Delta=[\sigma]$, and fix a face $\tau \in \Delta$ of dimension $d$. Extend the partial order on $[\tau]$ to a total order to get a sequence $\tau=\rho_{0}, \ldots, \rho_{N}=o$ so that $\left\{\rho_{0}, \ldots, \rho_{k}\right\}$ is closed in $[\tau]$ for every $0 \leq k \leq N$. We construct a sequence of objects $I_{0}^{\bullet}, \ldots, I_{N}^{\bullet}$, starting with $I_{0}^{\bullet}=N_{\tau}^{\bullet}$ and ending with $I_{N}=I_{\tau}^{\bullet}$, so that $I_{k}$ is an injective hull of $L_{\tau}^{\cdot}$ in the Serre subcategory of $\mathbf{P}_{\Phi}(\Delta)$ generated by $\left\{L_{\rho}^{\bullet} \mid \rho \in\left\{\rho_{1}, \ldots, \rho_{k}\right\} \cup(\Delta \backslash[\tau])\right\}$.

For the inductive step, assume that $I_{k-1}^{\bullet}$ has already been constructed. Set $\rho=\rho_{k}$. If $l \in \mathbb{Z}$, let $E_{l}=\operatorname{Ext}_{\mathbf{P}_{\Phi}(\Delta)}^{1}\left(N_{\rho}^{\bullet}\langle l\rangle, I_{k-1}^{\bullet}\right)$. The canonical element in $E_{l}^{*} \otimes E_{l}$ induces an element of $\operatorname{Ext}_{\mathbf{P}_{\Phi}(\Delta)}^{1}\left(E_{l} \otimes N_{\rho}^{\bullet}\langle l\rangle, I_{k-1}^{\bullet}\right)$. Combining these over all $l$ gives an element of

$$
\operatorname{Ext}_{\mathbf{P}_{\Phi}(\Delta)}^{1}\left(\bigoplus_{l \in \mathbb{Z}} E_{l} \otimes N_{\rho}^{\bullet}\langle l\rangle, I_{k-1}^{\bullet}\right) .
$$

Define $I_{k}^{\bullet}$ to be the corresponding extension object.

Since $\operatorname{Ext}_{\mathbf{P}_{\Phi}(\Delta)}^{1}=\operatorname{Hom}_{\mathbf{D}_{\Phi}(\Delta)}^{1}$, we can restate this as follows: $I_{k}$ is the object which fits into the distinguished triangle

$$
t\left(\operatorname{hom}_{\mathbf{D}_{\Phi}(\Delta)}^{\dot{x}_{\rho}}\left(N_{\dot{\rho}}^{\bullet}, I_{k-1}^{\bullet}\right)\right) \otimes N_{\rho}^{\bullet} \rightarrow I_{k-1}^{\bullet} \rightarrow I_{k}^{\bullet} \rightarrow \ldots,
$$

where $t$ is the truncation functor on bigraded vector spaces which keeps only elements of bidegree $(i, 1-i), i \in \mathbb{Z}$.

Comparing with Theorem 4.4.2 and using Lemma 4.2.6 gives Theorem 4.2.1(b).

\section{Connection with topology}

In this section, we construct the functor real ${ }_{\Delta}: \mathbf{D}(\Delta) \rightarrow D^{b}\left(X_{\Delta}\right)$ described in the Introduction, when $\Delta$ is a rational fan. We show that the combinatorial pushforward functors, stabilizations, etc., defined in Section 3 correspond under this functor to standard topological functors. In particular, we prove Theorems 1.4 .2 and 1.4 .3 from the Introduction.

Given an object $S^{\bullet} \in \mathbf{D}(\Delta)$, i.e. a complex of sheaves in $\operatorname{Inj}_{\Delta}$, we let real ${ }_{\Delta}\left(S^{\bullet}\right)$ be the single complex associated to the double complex $\Omega_{\Delta}^{\bullet}\left(S^{\bullet}\right)$, where $\Omega^{\bullet}$ is an additive functor from $\operatorname{Inj}_{\Delta}$ to the category $\operatorname{Kom}\left(X_{\Delta}\right)$ of complexes of $\mathbb{R}$-sheaves on $X_{\Delta}$. For this definition to satisfy the required properties, we need $\Omega_{\Delta}^{\bullet}\left(J_{\tau}\right)$ to be quasi-isomorphic to $i_{\tau *} \mathbb{R}_{O_{\tau}}$ for each $\tau$, and for the composition $I n j_{\Delta} \stackrel{\Omega_{\dot{\bullet}}^{\bullet}}{\longrightarrow}$ $\operatorname{Kom}\left(X_{\Delta}\right) \rightarrow D^{b}\left(X_{\Delta}\right)$ to be fully faithful.

There are several ways to produce such a functor. We use "controlled differential forms": forms which on a neighborhood of a point on a stratum $O_{\sigma}$ are isomorphic to a pullback by the canonical projection to $O_{\sigma}$. A similar construction was used by Goresky, Harder, and MacPherson [GHM] and Saper [Sa] to construct complexes on compactifications of locally symmetric spaces.

5.1. Toric geometry. We briefly recall the rudiments of toric geometry.

Let $V$ be a finite-dimensional $\mathbb{R}$-vector space spanned by a lattice $V_{\mathbb{Z}} \subset V$. Let $V_{\mathbb{Z}}^{*} \subset V^{*}$ be the dual lattice. For any $S \subset V^{*}$, let $S_{\mathbb{Z}}=S \cap V_{\mathbb{Z}}^{*}$. A rational cone $\sigma \subset V$ defines an affine toric variety $X_{[\sigma]}=\operatorname{Spec} A_{\sigma}$, where $A_{\sigma}$ is the ring $\bigoplus_{v \in \sigma^{\vee} \cap V_{\mathbb{Z}}^{*}} \mathbb{C} \phi_{v}$ with multiplication given by $\phi_{v} \phi_{v^{\prime}}=\phi_{v+v^{\prime}}$. 
If $\tau \prec \sigma$ are rational cones, we get a natural inclusion of rings $A_{\sigma} \rightarrow A_{\tau}$, which defines an open embedding $X_{[\tau]} \hookrightarrow X_{[\sigma]}$. For a rational fan $\Delta$ the associated toric variety $X_{\Delta}$ is the union of $X_{[\sigma]}, \sigma \in \Delta$, glued along these inclusions.

The torus $T=\operatorname{Hom}\left(V_{\mathbb{Z}}, \mathbb{C}^{*}\right)$ acts on $X_{\Delta}$, with one orbit $O_{\sigma}$ for each cone $\sigma \in \Delta$. A point $x$ lies in $O_{\sigma}$ if and only if $\phi_{v}(x) \neq 0 \Longleftrightarrow v \in V_{\sigma, \mathbb{Z}}^{\perp}$. Restricting from $\sigma_{\mathbb{Z}}^{\perp}$ to $V_{\sigma, \mathbb{Z}}^{\perp}$ gives an isomorphism $O_{\sigma} \cong \operatorname{Hom}\left(V_{\sigma, \mathbb{Z}}^{\perp}, \mathbb{C}^{*}\right) \cong\left(\mathbb{C}^{*}\right)^{\operatorname{codim} \sigma}$.

If $\tau \prec \sigma$, then there is a quotient map $p_{\tau, \sigma}: O_{\tau} \rightarrow O_{\sigma}$, given by

$$
\phi_{v}\left(p_{\tau, \sigma}(x)\right)=\left\{\begin{array}{c}
\phi_{v}(x), \quad v \in V_{\sigma, \mathbb{Z}}^{\perp}, \\
0, \quad v \in \sigma_{\mathbb{Z}}^{\vee} \backslash V_{\sigma, \mathbb{Z}}^{\perp} .
\end{array}\right.
$$

If $\rho \prec \tau \prec \sigma$, then $p_{\tau, \sigma} p_{\rho, \tau}=p_{\rho, \sigma}$.

If $\Delta$ is a quasifan contained in a fan $\Sigma$, then we define $X_{\Delta}$ to be $\bigcup_{\sigma \in \Delta} O_{\sigma}$, the union of $T$-orbits in $X_{\Sigma}$. This does not depend on the choice of $\Sigma$.

5.2. Realization functor. Let $\Delta$ be a rational quasifan in a vector space $V$. For each $\sigma \in \Delta$, the complex $\left(\Omega_{\sigma}^{\bullet}, d\right)$ of $\mathbb{R}$-valued $k$-forms on $O_{\sigma}$ is a fine resolution of the constant sheaf $\mathbb{R}_{O_{\sigma}}$. The torus $T$ acts on global sections of $\Omega_{\sigma}^{\bullet}$ by pullback; we will work with $T$-invariant forms $\Gamma\left(O_{\sigma}, \Omega_{\sigma}^{\bullet}\right)^{T}$.

The exponential map exp: $V / V_{\sigma} \otimes \mathbb{C} \rightarrow O_{\sigma}$, defined by $\phi_{x}(\exp (\xi))=e^{2 \pi \sqrt{-1}\langle\xi, v\rangle}$, is invariant under translations by the lattice $V_{\mathbb{Z}}$. Thus if we consider elements of $\Lambda_{\sigma}=\Lambda\left(V / V_{\sigma}\right)^{*}$ to be constant differential forms on $V / V_{\sigma}$, we get a map $\chi_{\sigma}: \Lambda_{\sigma} \rightarrow$ $\Gamma\left(O_{\sigma}, \Omega_{\sigma}^{\bullet}\right)$ whose image consists of $T$-invariant forms.

5.2.1. Lemma. $\chi_{\sigma}$ is injective. It induces a quasi-isomorphism of dg-algebras $\Lambda_{\sigma} \rightarrow$ $\Gamma\left(O_{\sigma}, \Omega_{\sigma}^{\bullet}\right)$, where the differential on $\Lambda_{\sigma}$ is trivial. If $\tau \prec \sigma$ are cones in $\Delta$, then

$$
p_{\tau, \sigma}^{*} \circ \chi_{\sigma}=\chi_{\tau} \circ \iota_{\tau, \sigma},
$$

where $\iota_{\tau, \sigma}: \Lambda_{\sigma} \rightarrow \Lambda_{\tau}$ is the natural homomorphism (i.e., the structure homomorphism for the sheaf $\Lambda_{\Delta}$ ).

Given a free graded $\Lambda_{\sigma}$-module $M$, define a complex of sheaves $\Omega_{\sigma}^{\bullet}(M)$ on $O_{\sigma}$ as follows. The space of sections of $\Omega_{\sigma}^{\bullet}(M)$ on an open set $U$ is $\Gamma\left(U, \Omega_{\sigma}^{\bullet}\right) \otimes_{\Lambda_{\sigma}} M$, where the (right) action of $\Lambda_{\sigma}$ on $\Gamma\left(U, \Omega_{\sigma}^{*}\right)$ is given by $\omega \lambda=\left.\omega \wedge \chi_{\sigma}(\lambda)\right|_{U}$. The grading is given by letting $\omega \otimes m$ have degree $i+j$ when $\omega$ is an $i$-form and $m \in M_{j}$. The boundary map is given by $d(\omega \otimes m)=d \omega \otimes m$. This is well defined since the previous lemma shows $d \circ \chi_{\sigma}=0$.

5.2.2. Lemma. If $M \cong \bigoplus_{j} \Lambda_{\sigma}\left[n_{j}\right]$, then $\Omega_{\sigma}^{\bullet}(M) \cong \bigoplus_{j} \Omega_{\sigma}^{\bullet}\left[n_{j}\right]$. It is a fine resolution of $\bigoplus_{j} \mathbb{R}_{O_{\sigma}}\left[n_{j}\right]$.

Let $\Lambda_{\Delta}$-mod $\operatorname{lf}_{\mathrm{lf}} \subset \Lambda_{\Delta}$-mod denote the full subcategory of locally free sheaves. Given $S \in \Lambda_{\Delta}-\bmod _{\mathrm{lf}}$, we will define a fine complex of sheaves $\Omega_{\Delta}^{\bullet}(S)$ on $X_{\Delta}$ by gluing the sheaves $\Omega_{\sigma}^{\bullet}\left(S_{\sigma}\right)$ for $\sigma \in \Delta$. Let us describe the sections of this complex on an open set $U \subset X_{\Delta}$. For any $\sigma \in \Delta$, put $U_{\sigma}=U \cap O_{\sigma}$.

If $\tau \prec \sigma$ are cones in $\Delta$, then we have a map

$$
q_{\tau, \sigma}: \Gamma\left(U_{\sigma}, \Omega_{\sigma}^{\bullet}\left(S_{\sigma}\right)\right) \rightarrow \Gamma\left(p_{\tau, \sigma}^{-1}\left(U_{\sigma}\right), \Omega_{\tau}^{\bullet}\left(S_{\tau}\right)\right),
$$

defined by $q_{\tau, \sigma}(\omega \otimes s)=p_{\tau, \sigma}^{*} \omega \otimes r_{\tau, \sigma}(s)$, where $r_{\tau, \sigma}: S_{\sigma} \rightarrow S_{\tau}$ is the structure homomorphism. Lemma 5.2 .1 ensures that this is well defined. It also clearly commutes with the differential $d$. 
Define sections of $\Omega_{\Delta}(S)$ on $U$ to be tuples

$$
\left(\alpha_{\sigma}\right) \in \bigoplus_{\sigma \in \Delta} \Gamma\left(U_{\sigma}, \Omega_{\sigma}^{\bullet}\left(S_{\sigma}\right)\right)
$$

which are "locally pullbacks" along the projections $p_{\tau, \sigma}$ : for any $\sigma \in \Delta$ and any point $x \in U_{\sigma}$, there must be a neighborhood $\widetilde{U}$ of $x$ contained in $U$, so that for any $\tau \prec \sigma$ the sections $\alpha_{\tau}$ and $q_{\tau, \sigma}\left(\alpha_{\sigma}\right)$ agree on $\widetilde{U} \cap p_{\tau, \sigma}^{-1}\left(U_{\sigma}\right)$. We define a boundary map on this complex by $d\left(\alpha_{\sigma}\right)=\left(d \alpha_{\sigma}\right)$.

5.2.4. Lemma. For any object $S \in \Lambda_{\Delta}-\bmod _{\mathrm{lf}}$, the sheaf $\Omega_{\Delta}^{\bullet}(S)$ is fine.

Proof. Sections of $\Omega_{\Delta}^{\bullet}(S)$ can be multiplied pointwise by sections of $\Omega_{\Delta}^{0}\left(\Lambda_{\Delta}\right)$, which are functions $f: X_{\Delta} \rightarrow \mathbb{R}$ which are locally pullbacks as defined above. There are partitions of unity of such functions subordinate to any open cover, so $\Omega_{\dot{\Delta}}^{\bullet}(S)$ is fine.

To define the functor real ${ }_{\Delta}$, assume first that we have $S^{\bullet} \in K^{b}\left(I n j_{\Delta}\right.$ ) (with no "half-grading"). Since $I n j_{\Delta}$ is a full subcategory of $\Lambda_{\Delta}-\bmod _{\text {lf }}$, we can let real ${ }_{\Delta}\left(S^{\bullet}\right)$ be the single complex coming from the double complex $\Omega_{\Delta}^{\bullet}\left(S^{\bullet}\right)$. A general object in $\mathbf{D}(\Delta)=K_{h}^{b}\left(\operatorname{Inj}_{\Delta}\right)$ is of the form $S^{\bullet}=S_{0}^{\bullet} \oplus S_{i}^{\bullet}\langle 1\rangle$, where each $S_{i}^{\bullet}$ is a complex in $\operatorname{Inj}_{\Delta}$; we let real $\Delta\left(S^{\bullet}\right)=\operatorname{real}_{\Delta}\left(S_{0}^{\bullet}\right) \oplus \operatorname{real}_{\Delta}\left(S_{\dot{\bullet}}\right)$.

Since $\Omega_{\Delta}^{\bullet}(S\{k\})=\Omega_{\Delta}^{\bullet}(S)[k]$ for any $S \in \Lambda_{\Delta}-\bmod _{l f}$ and $k \in \mathbb{Z}$, property (1) of Theorem 1.4.2 is obvious. It is also clear that real ${ }_{\Delta}$ takes values in $D_{u}^{b}\left(X_{\Delta}\right)$, the full subcategory of orbit-constructible objects in $D^{b}\left(X_{\Delta}\right)$ with unipotent monodromy, since $\operatorname{real}_{\Delta}\left(J_{\sigma}\right) \in D_{u}^{b}\left(X_{\Delta}\right)$ for every $\sigma \in \Delta$.

5.3. Functorial properties. Before proving parts (2)-(5) of Theorem 1.4.2, we prove two intermediate results. Fix an inclusion of rational quasifans $j: \Sigma \rightarrow \Delta$.

5.3.1. Lemma. (1) For any $S \in \Lambda_{\Delta}$ - $\bmod _{\mathrm{lf}}$, there is a natural isomorphism

$$
\Omega_{\Sigma}^{\bullet}\left(j^{*} S\right) \cong j^{*} \Omega_{\Delta}^{\bullet}(S) .
$$

(2) For any $S \in I n j_{\Sigma}$, there is a natural quasi-isomorphism

$$
\begin{aligned}
& \Omega_{\dot{\Delta}}^{\bullet}\left(j_{*} S\right) \stackrel{\sim}{\rightarrow} j_{*} \Omega_{\dot{\Sigma}}(S) . \\
\text { In particular, } \Omega_{\dot{\Delta}}\left(J_{\sigma}\right) & \cong j_{\sigma *} \Omega_{\dot{\sigma}}^{\bullet} .
\end{aligned}
$$

Proof. To see (1), note that the compatibility conditions among the components of a section (5.2.3) imply that the spaces of germs of sections of $\Omega_{\Delta}^{\bullet}(S)$ and of $\Omega_{\sigma}^{\bullet}\left(S_{\sigma}\right)$ at a point $x \in O_{\sigma}$ are isomorphic.

To construct the morphism for (2), consider an open set $U \subset X_{\Delta}$. The total space of sections of $j_{*} \Omega_{\Sigma}^{\bullet}(S)$ is $\Gamma\left(U \cap X_{\Sigma}, \Omega_{\Sigma}^{\bullet}(S)\right)$. This is given by tuples as in (5.2.3), satisfying the same compatibility condition, but where $\sigma$ runs over $\Sigma$ instead of $\Delta$. So if $\left(\alpha_{\sigma}\right)_{\sigma \in \Delta}$ is a section of $\Omega_{\Delta}^{\bullet}\left(j_{*} S\right)$ on $U$, forgetting $\alpha_{\sigma}$ for $\sigma \notin \Sigma$ provides the required section of $j_{*} \Omega_{\Sigma}^{\bullet}(S)$.

To show this is a quasi-isomorphism, it is enough to show it induces an isomorphism on stalk cohomology groups at every point $x \in X_{\Delta}$. Furthermore, we can assume that $S=J_{\sigma}$, and in fact that $\Sigma=\{\sigma\}$ and $S=\Lambda_{\sigma}$. Suppose that $x \in O_{\tau}$. Since $\Omega_{\Sigma}^{\bullet}(S)$ is a complex of fine sheaves, $j_{*} \Omega_{\Sigma}^{\bullet}(S)$ is isomorphic to the derived push-forward $R j_{*} \Omega_{\Sigma}^{\bullet}(S)$ in $D^{b}\left(X_{\Delta}\right)$. It follows that

$$
H^{\bullet}\left(j_{*} \Omega_{\Sigma}^{\bullet}(S)\right)_{x} \cong \Lambda\left(V_{\sigma}^{\perp} / V_{\tau}^{\perp}\right) .
$$


On the other hand, by part (1) of this lemma and Lemma 5.2 .2 we have

$$
H^{\bullet}\left(\Omega_{\Delta}\left(j_{*} S\right)\right)_{x} \cong \mathbb{R} \otimes_{\Lambda_{\tau}} S_{\tau}=\mathbb{R} \otimes_{\Lambda_{\tau}} \Lambda_{\sigma} .
$$

The induced map between these stalks is the obvious one induced by the quotient $V_{\sigma}^{\perp} \rightarrow V_{\sigma}^{\perp} / V_{\tau}^{\perp}$; it is an isomorphism.

\subsubsection{Corollary. If $J, J^{\prime} \in I_{n j_{\Delta}}$, then the natural map}

$$
\operatorname{Hom}_{\Lambda_{\Delta-\bmod }}\left(J, J^{\prime}\right) \rightarrow \operatorname{Hom}_{D^{b}\left(X_{\Delta}\right)}\left(\Omega_{\Delta}^{\bullet}(J), \Omega_{\Delta}^{\bullet}\left(J^{\prime}\right)\right)
$$

is an isomorphism (i.e. $\Omega_{\Delta}^{\bullet}$ is fully faithful on $\mathrm{Inj}_{\Delta}$ ).

Proof. It is enough to take $J=J_{\sigma}, J^{\prime}=J_{\tau}\{k\}$ for some $\sigma, \tau \in \Delta$ and $k \in \mathbb{Z}$. Applying $j_{\tau}^{*}$ to both sides and using the adjunction with $j_{\tau *}$, we can reduce to the case where $\Delta=\{\tau\}$ is a single cone and $J=\Lambda_{\tau}, J^{\prime}=\Lambda_{\tau}\{k\}$. In this case the right-hand side becomes

$$
\operatorname{Hom}_{D^{b}\left(X_{\Delta}\right)}\left(\Omega_{\tau}^{\bullet}, \Omega_{\tau}^{\bullet}[k]\right)=\operatorname{Hom}_{D^{b}\left(X_{\Delta}\right)}\left(\mathbb{R}_{O_{\tau}}, \mathbb{R}_{O_{\tau}}[k]\right)=H^{k}\left(O_{\tau}\right) .
$$

The left-hand side is $\Lambda^{k}\left(V_{\tau}^{\perp}\right)$, and the map sends $\lambda$ to exterior multiplication by $\chi_{\tau}(\lambda)$, acting on $\Omega_{\tau}^{\bullet}$. The result now follows from Lemma 5.2.1.

Part (2) of Theorem 1.4 .2 follows. The first two isomorphisms of part (3) of Theorem 1.4.2 (the ones for $j^{*}, j_{*}$ ) follow from Lemma 5.3.1

To show that $j_{!} \circ \mathrm{real}_{\Sigma} \simeq \mathrm{real}_{\Delta} \circ j_{\text {! }}$, note that Lemma 5.3.1(1) implies that $\operatorname{real}_{\Delta}\left(j_{! !} S^{\bullet}\right)$ is the extension by 0 of real $S_{\Sigma} S^{\bullet}$, where $j_{\text {!! }}$ is the combinatorial exten-

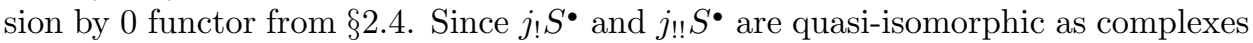
in $\Lambda_{\Delta}-\bmod _{l f}$, they remain quasi-isomorphic after applying real ${ }_{\Delta}$.

Finally we show that there is a functorial isomorphism

$$
\operatorname{real}_{\Sigma} \circ j ! S^{\bullet} \stackrel{\sim}{\rightarrow} j ! \circ \operatorname{real}_{\Delta} S^{\bullet},
$$

where $S^{\bullet} \in \mathbf{D}(\Delta)$. To construct the homomorphism, use the previous paragraph and the $j_{!}, j^{!}$adjunction, which holds in both $\mathbf{D}(\Delta)$ and $D^{b}\left(X_{\Delta}\right)$. It is easy to show it is an isomorphism when $S^{\bullet}=J_{\sigma}$; the general case follows by induction.

5.4. Stalks. Recall the functor $\Gamma_{\sigma}: \mathbf{D}(\Delta) \rightarrow D_{h}^{b}(\mathbb{R}$-mod) defined in 3.1 . We will show that it corresponds under real ${ }_{\Delta}$ to the stalk cohomology functor at any point $x \in O_{\sigma}$. First, define a "topological", nonmixed version of $\Gamma_{\sigma}$ :

$$
\Gamma_{\sigma}^{t o p}\left(S^{\bullet}\right)^{k}=\bigoplus_{p+q=k} \Gamma_{\sigma}\left(S^{\bullet}\right)_{q}^{p}
$$

5.4.1. Theorem. Take a cone $\sigma$ in the rational fan $\Delta$, and any point $x \in O_{\sigma}$. Let $i_{x}:\{x\} \rightarrow X_{\Delta}$ denote the inclusion. Then there is a natural isomorphism of graded vector spaces

$$
H^{\bullet}\left(i_{x}^{*} \operatorname{real}_{\Delta} S^{\bullet}\right) \stackrel{\sim}{\rightarrow} \Gamma_{\sigma}^{t o p}\left(S^{\bullet}\right) .
$$

This result, together with the results of the previous section, immediately implies Theorem 1.4.2, parts (4) and (5).

Proof. A cohomology class in $H^{k}\left(i_{x}^{*}\right.$ real $\left._{\Delta} S^{\bullet}\right)$ is represented by a germ of a section of $\Omega_{\Delta}^{k}\left(S^{\bullet}\right)$ at $x$ which is killed by the differential $d$. Such a germ is represented by a tuple $\left(\alpha_{\sigma}\right)$ of the form (5.2.3). Suppose $\alpha_{\tau}=\sum_{i=1}^{n} \omega_{i} \otimes s_{i}$, where each $\omega_{i}$ is a section of $\Omega_{\tau}^{\bullet}$, and $s_{i} \in S_{\tau}^{\bullet}$. Send this to $\sum_{i=1}^{n} \omega_{i}(x) \overline{s_{i}} \in \Gamma_{\tau}^{t o p}\left(S^{\bullet}\right)^{k}$, where $\omega(x)$ is 
the value of $\omega$ at $x$ if $\omega$ is a 0 -form, and is zero otherwise, and $s \mapsto \bar{s}$ is the natural quotient map $S_{\tau}^{\bullet} \rightarrow \mathbb{R} \otimes_{\Lambda_{\tau}} S_{\tau}$.

It is easy to check that this is a well-defined map, and that it passes to cohomology, giving a map (5.4.2). To check that it is an isomorphism it is enough to prove the case $S^{\bullet}=j_{\sigma !} \Lambda_{\sigma}$, which is easy: the stalk at a point of $O_{\tau}$ is $\mathbb{R}$ if $\tau=\sigma$ and 0 otherwise.

5.5. $\Phi$-stable sheaves. We now prove Theorem 1.4.3, which gives the topological interpretation of combinatorial completions and the stable category $\mathbf{D}_{\Phi}(\Delta)$. Our first goal is to prove the "only if" direction of part (1) of this theorem: that objects of $\mathbf{P}_{\Phi}(\Delta)$ are sent into $\mathcal{P}_{\Phi}\left(X_{\Delta}\right)$ by real ${ }_{\Delta, \Phi}$. To do this, we must study how our categories of combinatorial and topological sheaves are affected by changing the ambient vector space of a fan.

Let $\Sigma$ be a rational quasifan in $V$, and suppose that every cone of $\Sigma$ is contained in a rational subspace $W \subset V$. For each $\sigma \in \Sigma$, we denote by $\hat{\sigma}$ the same cone, but thought of as a subset of the ambient space $W$ instead of $V$. Let $\widehat{\Sigma}$ be the fan $\{\hat{\sigma} \mid \sigma \in \Delta\}$. The vector space span of the cones has not changed: we have $W_{\hat{\sigma}}=V_{\sigma}$. But the sheaf of rings $\Lambda_{\widehat{\Sigma}}$ is different, since $\left(\Lambda_{\widehat{\Sigma}}\right)_{\hat{\sigma}}=\Lambda_{\hat{\sigma}}=\Lambda\left(W_{\hat{\sigma}}^{\perp}\right)$, where $W_{\hat{\sigma}}^{\perp}$ is the annihilator of $W_{\hat{\sigma}}$ in $W$.

Now suppose $Z \subset V^{*}$ is a rational subspace complementary to the annihilator $W^{\perp}$ of $W$ in $V^{*}$. The composition $Z \hookrightarrow V^{*} \rightarrow W^{*}$ is an isomorphism, so we get an inclusion $\iota: W^{*} \rightarrow Z \rightarrow V^{*}$. For any $\sigma \in \Sigma$ we have $\iota\left(\hat{\sigma}^{\vee}\right) \subset \sigma^{\vee}$, so we get a morphism $\pi_{Z}: X_{\Sigma} \rightarrow X_{\widehat{\Sigma}}$, defined by $\phi_{v}\left(\pi_{Z}(x)\right)=\phi_{R(v)}(x)$ (here the toric variety $X_{\widehat{\Sigma}}$ is defined with respect to the lattice $\left.W_{\mathbb{Z}}^{*}=\iota^{-1}\left(V_{\mathbb{Z}}^{*}\right) \subset W^{*}\right)$. It is a fiber bundle whose fibers are tori $\left(\mathbb{C}^{*}\right)^{\operatorname{dim} V-\operatorname{dim} W}$.

Next, we define the analogue for combinatorial sheaves of the functor $\pi_{Z}^{*}$. We have inclusions $\Lambda_{\hat{\sigma}} \rightarrow \Lambda_{\sigma}, \sigma \in \Sigma$, which are compatible with the structure maps of the sheaves $\Lambda_{\Sigma}$ and $\Lambda_{\widehat{\Sigma}}$. Thus we can define a functor $G_{Z}: \Lambda_{\widehat{\Sigma}}$-mod $\rightarrow \Lambda_{\Delta}$-mod by $G_{Z}(S)_{\sigma}=\Lambda_{\sigma} \otimes_{\Lambda_{\hat{\sigma}}} S_{\sigma}$. It induces a triangulated functor $\mathbf{D}(\widehat{\Sigma}) \rightarrow \mathbf{D}(\Sigma)$, which we again denote by $G_{Z}$.

5.5.1. Proposition. There is a natural quasi-isomorphism

$$
\pi_{Z}^{*} \circ \operatorname{real}_{\widehat{\Sigma}} \simeq \operatorname{real}_{\Sigma} \circ G_{Z}
$$

of functors $\mathbf{D}(\widehat{\Sigma}) \rightarrow D^{b}\left(X_{\Sigma}\right)$.

Proof. First consider the case where $\Sigma=\{\sigma\}$ is a single cone, so $X_{\Sigma}=O_{\sigma}, X_{\widehat{\Sigma}}=$ $O_{\hat{\sigma}}$. Take an object $M \in \Lambda_{\Delta}-\bmod _{\mathrm{lf}}$, i.e. a free $\Lambda_{\hat{\sigma}}$-module. Define a homomorphism

$$
\pi_{Z}^{*} \Omega^{\bullet}(M) \rightarrow \Omega^{\bullet}\left(G_{Z}(M)\right)
$$

as follows. A section of $\pi_{Z}^{*} \Omega^{\bullet}(M)$ will be of the form $\pi^{*} \omega \otimes m$ for $\omega$ a section of $\Omega_{\hat{\sigma}}$ and $m \in M$. Send this to $\pi^{*} \omega \otimes(1 \otimes m)$, which is a section of $\Omega^{\bullet}\left(G_{Z}(M)\right)=$ $\Omega^{\bullet}\left(\Lambda_{\sigma} \otimes_{\Lambda_{\hat{\sigma}}} M\right)$. The fact that this is well defined follows from the identity

$$
\chi_{\sigma} \circ \iota=\pi_{Z}^{*} \circ \chi_{\hat{\sigma}},
$$

which is essentially Lemma 5.2.1 in another guise. To show that it is an isomorphism, it is enough to consider $M=\Lambda_{\hat{\sigma}}$, in which case it follows from (5.5.3) and Lemma 5.2.1

For general $\Sigma$, the homomorphism (5.5.2) is defined componentwise by the same formula. To show it is an isomorphism, use the case $\Sigma=\{\sigma\}$ and Lemma 5.3.1. 
Let $\Phi$ be a rational completion on a quasifan $\Delta$. Take a cone $\sigma \in \Delta$, and let $\Sigma=[\sigma] \cap \Delta$. Let $W=V_{\sigma}, Z=\Phi_{\sigma}$. The previous discussion gives a fan $\widehat{\Sigma}$ in $W$ and a map $\pi=\pi_{\sigma}: X_{\Sigma} \rightarrow X_{\widehat{\Sigma}}$; this is the map used in Definition 1.3.2 to define the category $\mathcal{P}_{\Phi}\left(X_{\Delta}\right)$.

5.5.4. Proposition. There is a functor $Q: \mathbf{D}_{\Phi}(\Sigma) \rightarrow \mathbf{D}(\widehat{\Sigma})$ so that $G_{Z} \circ Q$ is naturally isomorphic to the forgetful functor $\mathbf{D}_{\Phi}(\Sigma) \rightarrow \mathbf{D}(\Sigma)$.

Proof. Suppose $\left(S,\left\{S_{\tau}^{\natural}\right\}\right) \in \Lambda_{\Delta}-\bmod _{\Phi}$. If $\hat{\tau} \in \widehat{\Sigma}$, define $Q(S)_{\hat{\tau}}=\Lambda_{\hat{\tau}} S_{\sigma}^{\natural} \subset S_{\sigma}$. Since $\Lambda_{\hat{\tau}}=\Lambda\left(\Phi_{\sigma}^{\tau}\right)$, these stalks fit together to give an object in $\Lambda_{\widehat{\Sigma}}$-mod.

5.5.5. Corollary. If $S^{\bullet} \in \mathbf{D}_{\Phi}(\Delta)$, then $\operatorname{real}_{\Delta, \Phi}\left(\left.S^{\bullet}\right|_{X_{\Sigma}}\right) \cong \pi^{*}$ real $\widehat{\Sigma}_{\widehat{S}} \widehat{S}$ for some object $\widehat{S} \bullet \in \mathbf{D}(\widehat{\Sigma})$.

Proof. Take $\widehat{S}^{\bullet}=Q\left(\left.S^{\bullet}\right|_{X_{\Delta}}\right)$ and use Propositions 5.5.1 and 5.5.4,

Theorem 1.4.3 (1) follows.

5.6. Ext's and injectives. Finally, we prove the remaining parts of Theorem 1.4.3. To simplify notation, we will indicate the result of applying real ${ }_{\Delta, \Phi}$ by a change to script letters: $\mathcal{L}_{\sigma}=\operatorname{real}_{\Delta, \Phi}\left(L_{\sigma}^{\bullet}\right), \mathcal{I}_{\sigma}=\operatorname{real}_{\Delta, \Phi}\left(I_{\sigma}^{\bullet}\right)$, etc. By the results of the previous section, these objects lie in $\mathcal{P}_{\Phi}\left(X_{\Delta}\right)$.

We begin with a special case of Theorem $1.4 .3(3)$. Define a bifunctor $\operatorname{ext}_{\mathbf{P}_{\Phi}(\Delta)}^{1}$ by

$$
\operatorname{ext}_{\mathbf{P}_{\Phi}(\Delta)}^{1}\left(A^{\bullet}, B^{\bullet}\right)=\bigoplus_{k \in \mathbb{Z}} \operatorname{Ext}_{\mathbf{P}_{\Phi}(\Delta)}^{1}\left(A^{\bullet}, B^{\bullet}\langle k\rangle\right) .
$$

5.6.1. Proposition. Suppose $S^{\bullet} \in \mathbf{P}_{\Phi}(\Delta)$ and $\sigma \in \Delta$. Then the map

$$
\operatorname{ext}_{\mathbf{P}_{\Phi}(\Delta)}^{1}\left(L_{\sigma}^{\bullet}, S^{\bullet}\right) \rightarrow \operatorname{Ext}_{\mathcal{P}_{\Phi}\left(X_{\Delta}\right)}^{1}\left(\mathcal{L}_{\sigma}, \mathcal{S}\right)
$$

induced by $\mathrm{real}_{\Phi, \Delta}$ is an isomorphism.

Assuming this for the moment, we obtain

5.6.3. Corollary. $\mathcal{I}_{\sigma}$ is an injective hull of $\mathcal{L}_{\sigma}$.

Proof. Taking $S^{\bullet}=I_{\sigma}^{\bullet}$ in Proposition 5.6 .1 shows that $\operatorname{Ext}^{1}\left(\mathcal{L}_{\tau}, \mathcal{I}_{\sigma}\right)=0$ for all $\tau \in \Delta$, so $\mathcal{I}_{\sigma}$ is injective. Applying real ${ }_{\Delta, \Phi}$ to the injection $L_{\sigma}^{\bullet} \rightarrow I_{\sigma}^{\bullet}$ gives an injection $\mathcal{L}_{\sigma} \rightarrow \mathcal{I}_{\sigma}$. To see that $\mathcal{I}_{\sigma}$ is indecomposable, note that $\mathbf{P}_{\Phi}(\Delta)$ and $\mathcal{P}_{\Phi}\left(X_{\Delta}\right)$ are full subcategories of $\mathbf{P}(\Delta)$ and $\mathcal{P}\left(X_{\Delta}\right)$, respectively, and hence full subcategories of $\mathbf{D}(\Delta)$ and $D^{b}\left(X_{\Delta}\right)$. So using Theorem 1.4.2(2), we see that if $\mathcal{I}_{\sigma}$ were decomposable, there would be a nontrivial idempotent in the ring

$$
\bigoplus_{k \in \mathbb{Z}} \operatorname{Hom}_{\mathbf{P}_{\Phi}(\Delta)}\left(I^{\bullet}, I^{\bullet}\langle k\rangle\right) \text {. }
$$

Such an idempotent must actually lie in $\operatorname{Hom}_{\mathbf{P}_{\Phi}(\Delta)}\left(I^{\bullet}, I^{\bullet}\right)$, contradicting the indecomposability of $I^{\bullet}$.

This implies part (3) of Theorem 1.4.3, given $S_{1}, S_{2}^{\bullet} \in \mathbf{D}_{\Phi}(\Delta)$, take injective resolutions and apply Theorem $1.4 .2(2)$ again.

We can now deduce the "if" direction of Theorem[1.4.3(1). Suppose $S^{\bullet} \in \mathbf{P}(\Delta)$, and $\mathcal{S}=\operatorname{real}_{\Delta} S^{\bullet}$ is in $\mathcal{P}_{\Phi}(\Delta)$. Since $\mathcal{P}_{\Phi}(\Delta)$ has enough injectives by the previous corollary, there is an injection $\phi: \mathcal{S} \rightarrow \mathcal{I}$, where $\mathcal{I} \in \mathcal{P}_{\Phi}(\Delta)$ is injective; say $\mathcal{I}=\operatorname{real}_{\Delta, \Phi} I^{\bullet}$, where $I^{\bullet}$ is injective in $\mathbf{P}_{\Phi}(\Delta)$. Replacing $I^{\bullet}$ by $\bigoplus_{k=-K}^{K} I^{\bullet}\langle k\rangle$ if 
necessary, we can construct $f \in \operatorname{Hom}_{\mathbf{D}(\Delta)}\left(S^{\bullet}, I^{\bullet}\right)$ which is sent to $\phi$ by real $\Delta$. Since $\phi$ is an injection, so is $f$. We have embedded $S^{\bullet}$ into an object from $\mathbf{P}_{\Phi}(\Delta)$, so $S \bullet \in \mathbf{P}_{\Phi}(\Delta)$ also.

Finally, we prove Proposition 5.6.1. Consider the commutative square

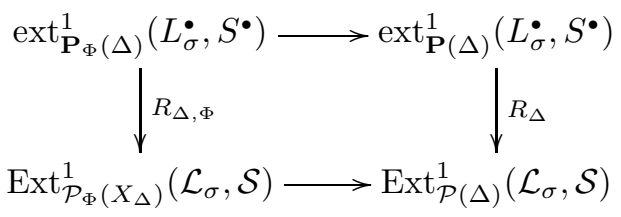

Here $R_{\Delta, \Phi}$ is the map (5.6.2), the other vertical map $R_{\Delta}$ is defined similarly, and the horizontal maps are the injections induced from the forgetful functors. We call an element of $\operatorname{ext}_{\mathbf{P}(\Delta)}^{1}\left(L_{\sigma}^{\bullet}, S^{\bullet}\right)$ " $\Phi$-stable" if it is in the image of the upper horizontal map.

To show $R_{\Delta, \Phi}$ is injective, it is enough to show that $R_{\Delta}$ is injective. This follows from Theorem $1.4 .2(2)$, since $\operatorname{Ext}_{\mathbf{P}(\Delta)}^{1} \rightarrow \operatorname{Hom}_{\mathbf{D}(\Delta)}^{1}$ and $\operatorname{Ext}_{\mathcal{P}\left(X_{\Delta}\right)}^{1} \rightarrow \operatorname{Hom}_{D^{b}\left(X_{\Delta}\right)}^{1}$ are isomorphisms of bifunctors.

To show that $R_{\Delta, \Phi}$ is surjective, take $\psi \in \operatorname{Ext}_{\mathcal{P}_{\Phi}\left(X_{\Delta}\right)}^{1}\left(\mathcal{L}_{\sigma}, \mathcal{S}\right)$, with image $\phi \in$ $\operatorname{Ext}_{\mathcal{P}\left(X_{\Delta}\right)}^{1}\left(\mathcal{L}_{\sigma}, \mathcal{S}\right)$. There exists $\tilde{\phi} \in \operatorname{ext}_{\mathbf{P}(\Delta)}^{1}\left(L_{\sigma}^{\bullet}, S^{\bullet}\right)$ so that $R_{\Delta}(\tilde{\phi})=\phi$. We must show that $\tilde{\phi}$ is $\Phi$-stable.

To do this, use the setup of Proposition [5.5.4 let $\Sigma=\Delta \cap[\sigma]$, let $\widehat{\Sigma}$ be the same quasifan with the ambient space $W=V_{\sigma}$, and let $G=G_{\Phi_{\sigma}}: \mathbf{D}(\widehat{\Sigma}) \rightarrow \mathbf{D}(\Sigma)$. The shifted functor $G^{\prime}=G[\operatorname{codim} \sigma]$ preserves perversity.

5.6.4. Lemma. A map $\tilde{\phi} \in \operatorname{ext}_{\mathbf{P}(\Delta)}^{1}\left(L_{\sigma}^{\bullet}, S^{\bullet}\right)$ is $\Phi$-stable if and only if the restriction $\left.\tilde{\phi}\right|_{\Sigma}$ is in the image of $G^{\prime}: \operatorname{ext}_{\mathbf{P}(\widehat{\Sigma})}^{1}\left(L_{\sigma}^{\bullet}, S^{\bullet}\right) \rightarrow \operatorname{ext}_{\mathbf{P}(\Sigma)}^{1}\left(L_{\sigma}^{\bullet}, S^{\bullet}\right)$.

Proof. The "only if" direction follows from Corollary 5.5.5. For the other direction, note that for any $\tau \in \Delta$ we have $i_{\tau}^{!} S^{\bullet} \in \mathbf{D}^{\geq 0}(\Delta)$ and $i_{\tau}^{*} L_{\sigma}^{\bullet} \in \mathbf{D}^{\leq a}(\Delta)$, where $a=0$ if $\tau=\sigma$ and $a=-1$ if $\tau \neq \sigma$. As in the proof of Proposition 3.5.1, the spectral sequences (3.5.2) and (3.5.3) imply that if $\tau \neq \sigma$, then the map

$$
\operatorname{hom}_{\mathbf{P}_{\Phi}(\Delta)}^{k}\left(i_{\tau}^{*} L_{\sigma}^{\bullet}, i_{\tau}^{!} S^{\bullet}\right) \rightarrow \operatorname{hom}_{\mathbf{P}(\Delta)}^{k}\left(i_{\tau}^{*} L_{\sigma}^{\bullet}, i_{\tau}^{!} S^{\bullet}\right)
$$

is an isomorphism for $k=1$, an injection for $k>1$, and both sides vanish if $k \leq 0$, while if $\tau=\sigma$, it is an isomorphism when $k=0$, an injection if $k>0$, and both sides vanish if $k<0$.

As a result, in order for $\tilde{\phi}$ to be $\Phi$-stable, it is enough for $\left.\tilde{\phi}\right|_{\Sigma}$ to be $\Phi$-stable. But $\left.L_{\sigma}^{\bullet}\right|_{\Sigma}$ has support only on $\sigma$, where it is a shifted copy of $\Lambda_{\sigma}$. So $\Phi$-stability of $\left.\tilde{\phi}\right|_{\Sigma}$ is equivalent to $\left.\tilde{\phi}\right|_{\Sigma}$ being in the image of $G^{\prime}$.

Now, since $\phi$ comes from an extension in $\mathcal{P}_{\Phi}\left(X_{\Delta}\right),\left.\phi\right|_{X_{\Sigma}}$ is in the image of

$$
\pi^{*}: \operatorname{ext}_{\mathcal{P}\left(X_{\widehat{\Sigma}}\right)}^{1}\left(\widehat{\mathcal{L}}_{\sigma}, \widehat{\mathcal{S}}\right) \rightarrow \operatorname{ext}_{\mathcal{P}\left(X_{\Sigma}\right)}^{1}\left(\mathcal{L}_{\sigma}, \mathcal{S}\right)
$$

for some $\widehat{\mathcal{S}}$ and $\widehat{\mathcal{L}}_{\sigma} \in \mathcal{P}\left(X_{\widehat{\Sigma}}\right)$. It follows, using Proposition 5.5.1 and Theorem $1.4 .2(2)$ that $\left.\tilde{\phi}\right|_{\Sigma}$ is in the image of $G^{\prime}$, so we can apply the previous lemma to complete the proof of Proposition 5.6.1. 


\section{ACKNOWLEDGMENTS}

This paper has had a long gestation, and there are many people who should be thanked. At various points conversations with R. MacPherson, A. Beilinson, V. Ginzburg, W. Soergel, R. Bezrukavnikov, G. Barthel, J.-P. Brasselet, K.-H. Fieseler, L. Kaup, R. Stanley, K. Vilonen, I. Mirkovic, and V. Lunts have been invaluable.

\section{REFERENCES}

[BBFK] G. Barthel, J.-P. Brasselet, K.-H. Fieseler, and L. Kaup, Combinatorial intersection cohomology for fans, Tohoku Math. J. (2) 54 (2002), no. 1, 1-41. MR.1878925 (2003a:14032)

[BaBo] V. Batyrev and L. Borisov, Mirror duality and string-theoretic Hodge numbers, Invent. Math. 126 (1996), no. 1, 183-203. MR.1408560 (97k:14039)

[BBD] A. Beilinson, J. Bernstein, and P. Deligne, Faisceaux pervers, Analysis and topology on singular spaces, I (Luminy, 1981), 5-171, Astérisque 100, Soc. Math. France, Paris, 1982. MR0751966 (86g:32015)

[BGSch] A. Beilinson, V. Ginsburg, and V. Schechtman, Koszul duality, J. Geom. Phys. 5 (1988), no. 3, 317-350. MR.1048505 (91c:18011)

[BGS] A. Beilinson, V. Ginzburg, and W. Soergel, Koszul duality patterns in representation theory, J. Amer. Math. Soc. 9 (1996), no. 2, 473-527. MR.1322847(96k:17010)

[BGG] J. Bernstein, I. Gelfand, and S. Gelfand, Algebraic vector bundles on $P^{n}$ and problems of linear algebra, Func. Anal. App. 12 (1978), no. 3, 66-67. MR0509387 (80c:14010a)

[BL] T. Braden and V. Lunts, Equivariant-constructible Koszul duality for dual toric varieties, Adv. Math. 201 (2006), no. 2, 408-453. MR2211534

[BrL] P. Bressler and V. Lunts, Intersection cohomology on nonrational polytopes, Compositio Math. 135 (2003), no. 3, 245-278. MR1956814 (2004b:52016)

[Boe] B. Boe, A counterexample to the Gabber-Joseph conjecture, Contemp. Math. 139 (1992), 1-3. MR1197826 (94a:22032)

[F] W. Fulton, Mathematics Studies, 131, Princeton University Press, 1993. MR.1234037 (94g:14028)

[GHM] M. Goresky, G. Harder, and R. MacPherson, Weighted cohomology, Invent. Math. 116 (1994), no. 1-3, 139-213. MR1253192 (95c:11068)

[GKM] M. Goresky, R. Kottwitz, and R. MacPherson, Equivariant cohomology, Koszul duality and the localization theorem, Invent. Math. 131 (1998), no. 1, 25-83. MR1489894 (99c:55009)

[GM] S. Gelfand and Y. Manin, Methods of homological algebra, Springer-Verlag, Berlin, 1996. MR.1438306 (97j:18001)

[G] V. Ginsburg, Perverse sheaves and $\mathbb{C}^{*}$ actions, J. Amer. Math. Soc. 4 (1991), 483-490. MR.1091465 (92d:14013)

[Ka] K. Karu, Hard Lefschetz Theorem for Nonrational Polytopes, Invent. Math. 157 (2004), no. 2, 419-447. MR2076929

[KS] M. Kashiwara and P. Schapira, Sheaves on Manifolds, Springer 1990. MR1074006 (92a:58132)

[KL] D. Kazhdan and G. Lusztig, Representations of Coxeter groups and Hecke algebras, Invent. Math. 53 (1979), 165-184. MR0560412 (81j:20066)

[L] V. Lunts, Equivariant sheaves on toric varieties, Compositio Math. 96 (1995), no. 1, 63-83. MR.1323725 (96e:14060)

[M] M. McConnell, Intersection Cohomology of Toric Varieties, preprint, 1999.

[MV] R. MacPherson and K. Vilonen, Elementary construction of perverse sheaves, Invent. Math. 84 (1986), 403-435. MR0833195 (87m:32028)

[Sa] L. Saper, L-modules and micro-support, preprint math.RT/0112251, 2001, 88 pp.

[Sa2] L. Saper, On the cohomology of locally symmetric spaces and of their compactifications, Current developments in mathematics, 2002, 219-289, Int. Press, Somerville, MA, 2003. MR2062320 (2005g:32043) 
[SSV] B. Shapiro, M. Shapiro, and A. Vainshtein, On combinatorics and topology of pairwise intersections of Schubert cells in $\mathrm{SL}_{n} / \mathcal{B}$, The Arnold-Gelfand mathematical seminars, 397-437, Birkhäuser Boston, Boston, MA, 1997. MR1429903 (98e:14053)

[S] W. Soergel, Kategorie $\mathcal{O}$, perverse Garben und Moduln über den Koinvarienten zur Weylgruppe, J. Amer. Math. Soc. 3 (1990), no. 2, 421-445. MR1029692 (91e:17007)

[St] R. Stanley, Subdivision and local h-vectors, J. Amer. Math. Soc. 5 (1992), 805-851. MR.1157293 (93b:52012)

[V] J.-L. Verdier, Prolongement des faisceaux pervers monodromiques, Differential systems and singularities (Luminy, 1983), Astérisque 130 (1985), 218-236. MR0804055 (87d:32019)

[W] A. Weber, Weights in the cohomology of toric varieties, Cent. Eur. J. Math. 2 (2004), no. 3, 478-492 (electronic). MR2113544

Department of Mathematics and Statistics, University of Massachusetts, Amherst, MASSACHUSETTS 01003

E-mail address: braden@math.umass.edu 